\title{
Classification Software Engineering Documents Based on Hybrid Model
}

\author{
Nada N. Saleem \\ Rasha Gh. Saeed \\ Nada_N_S@uomosul.edu.iq \\ College of Computer Sciences and Mathematics \\ University of Mosul, Mosul, Iraq
}

\section{Received on: 16/07/2018}

Accepted on: 27/08/2018

Care about automated documents classification has increased since the appearance of the digital documents and the wide diffusion of Internet. In the 1990's, the computer performance has greatly improved and has led to the methods of machine learning to establish automated classifiers. These methods have achieved good speed and classification's accuracy and researchers still investigate in this field to accomplish more accuracy and less time. Artificial immunologic systems have shown high performance in such as data clustering and anomaly detection which can be ascribed to the nature of the immunologic system in protecting the body.

Some of the present methods and ways used in the training process of the document classification are time consuming and others have less accuracy rate concerned with the classification of the related document as software engineering document classes. For these reasons, this research deals with the study of Natural Immune System and using the dynamic process of the Adaptive Immune System work by hybridization Negative Selection (NS) and Positive Selection (PS) techniques and to propose a hybrid model called the Hybrid Positive Negative Selection Model (HPNS) to classify Software Engineering documents as they comprise information related to developing the software systems, that makes it easy for the software engineer who works in maintenance.

HPNS has high classification's speed and accuracy besides easy and flexible use by designing interfaces that make it easy for the user to deal with the system. In order to improve the quality and the efficiency of HPNS method, it was compared to one of the best and wellknown methods of classification referred to as, Naive Bayes(NB). After conducting several experiments on a various group of software engineering documents, evaluations results have shown that the accuracy of the Adaptive immunologic method (HPNS) has reached (HPNS) (95\%), whereas Naïve classification method has reached (90\%) with training and classification speed that doesn't exceed one minute. This shows the feasibility of using the algorithms of AIS systems in the field of information recovery and documents classification. This system was built and programmed in Java language and was implemented under an operating system environment Microsoft Windows7.

Keyword: Artificial immunologic systems, Negative Selection, Positive Selection, Software Engineering Documents, Documents Classification.

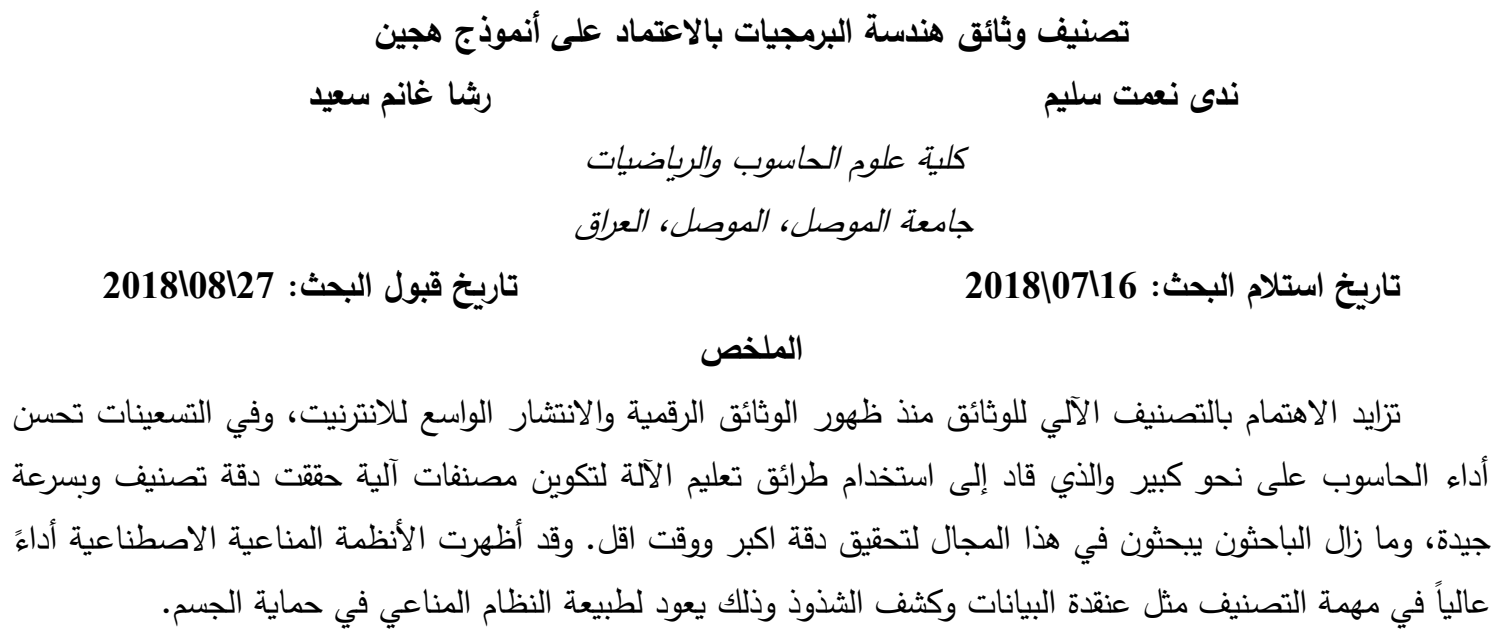


إن بعض الطرائق والأساليب الحالية تحتاج إلى وقت في عملية التدريب لتصنيف الوثائق والبعض الآخر من الأساليب

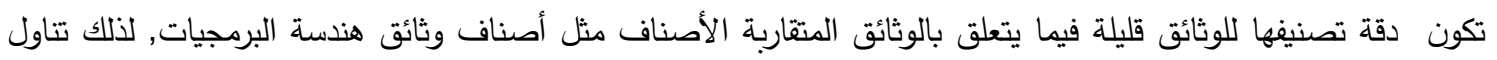

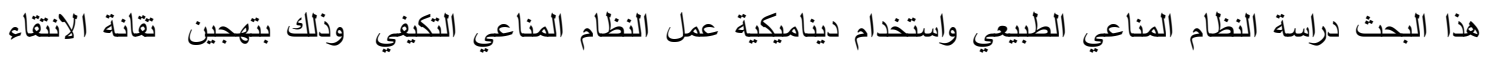

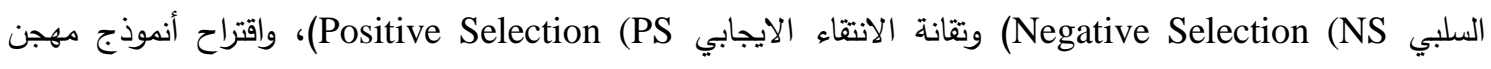
المسمى أنموذج الانتقاء الإيجابي والسلبي (Nodel(HPNS) لتصنيف وثنائق هندسة البرمجيات لما تحويه من معلومات متعلقة بتطوير الأنظمة البرمجية التي تسهل العمل على نحو كبير على مهندس البرمجيات الذي يقوم بالتطوير والصيانة.

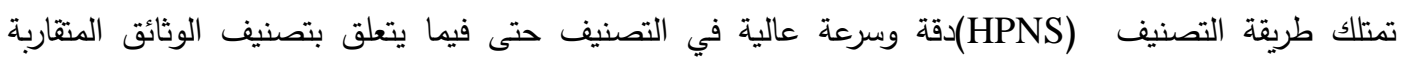
الأصناف ولبرهنة فاعلية وجودة طريقة) قورنت بأحد أفضل وأشهر طرائق التصنيف المستخدمة وهي طريقة Naïve Bayes (NB)

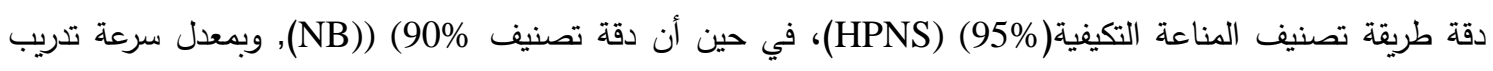

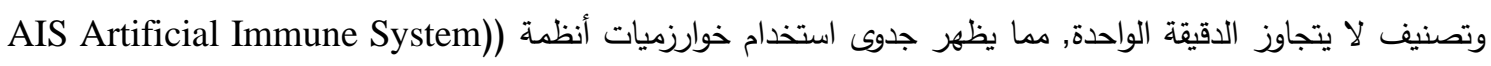
في حقل استرجاع المعلومات وتصنيف الوثائق. بني هذا الأنموذج وبرمجته بلغة جافا وتتفيذه تحت بئنة بئة نظام تثغيل

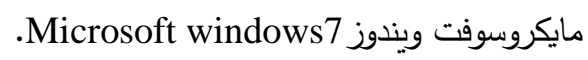
الكلمات المفتاحية: الأنظة المناعية الاصطناعية، الانتقاء السلبي, الانتقاء الإيجابي، تصنيف الوثائق، وثائق هندسة البرمجيات.

1. مقدمة

أصبح تصنيف الوثائق الآلي مجال بحث وتطبيق مهم منذ ظهور الوثائق الرقمية؛ إذ يعد تصنيف النص

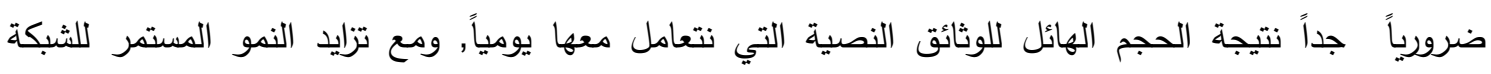

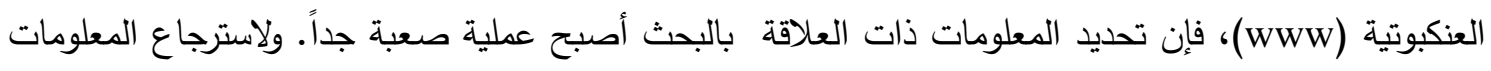
بأقل وقت ممكن وبأعلى درجة من العلاقة جاء دور التصنيف الآلي؛ إذ إن تصنيف الثبكة الآلي يساعد في

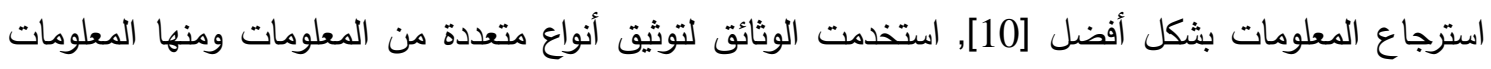

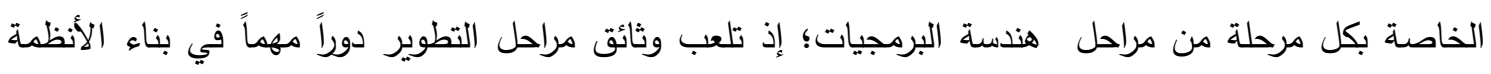
البرمجية، ويشكل التوثيق وسيلة لتقديم الرؤية ضمن عملية البرمجيات، ويعد دور الاتصال أساسيا لتطوير

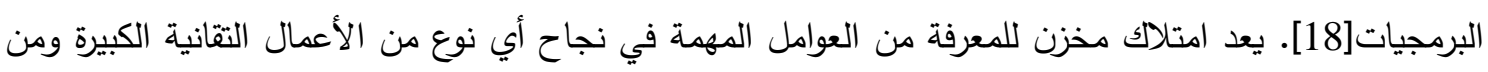
الضروري تسجيل تلك المعرفة في الوثائق؛ إذ تكون الوثائق بصيغة الكترونية مدكن البحث عنها ومشاركتها

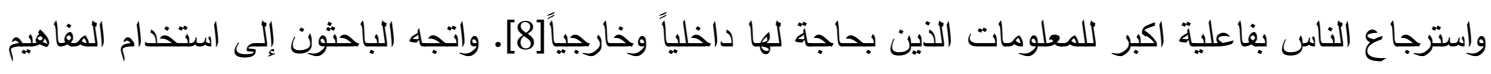

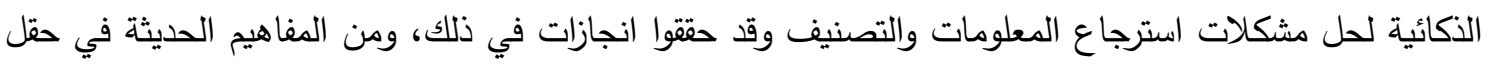

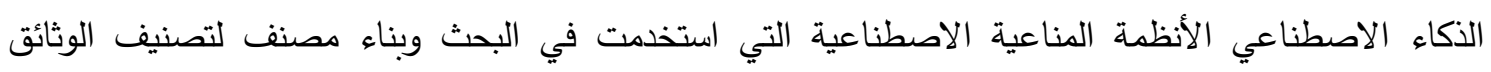
لمراحل هندسة البرمجيات.

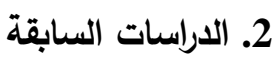

حاول الباحثون في السنوات الماضية إيجاد حلول واقتراحات كثيرة لتصنيف الوثائق بطرائق سهلة، سريعة، ذات

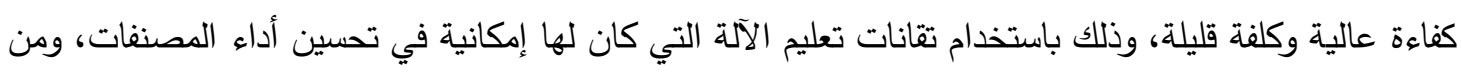
هذه البحوث: 
1. في عام 1997 قدم الباحثان Pazzani و Bنثاً عن عدد من خوارزميات التعلم المختلفة, واختبار

أداء ودقة مصنف Naïve Bayes (NB) ، والمجاور الأقرب، واشجار القرار والثبكات العصبية ووجد أن

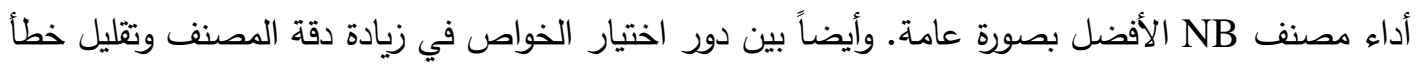

التصنيف [31].

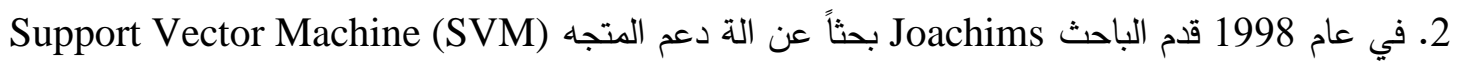
بوصفها طريقة لتصنيف الوثائق؛ إذ أثبتت هذه الطريقة كفاءتها بالتصنيف إلا أنها تعاني من التعقيد، ويقع

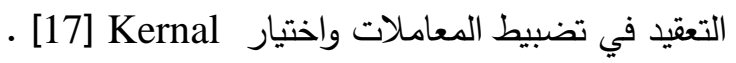

3. في عام 1999 قدم الباحثان Cohen و Singer بحثاً لطرائق التعليم لتصنيف النص واستخدما أشجار القرار وبينًا قابليتها في التصنيف؛ إذ تمتاز بسهولة الفهر وتقلل تعقيد المشكلة إلا أن أشجار القرار تتطلب وقت تدان تدريب أطول، كما أن الوثيقة تُمثل في هيكلية شجرة، وفي هذه الحالة عند حصول خطأ في مستوى عالٍ فإن أي شجرة

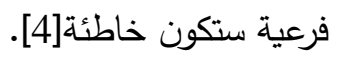

4. في عام 2002 قدم الباحث Sebastiani بحثاً عن طرائق التصنيف واستخدام الثبكة العصبية وامكانيتها في

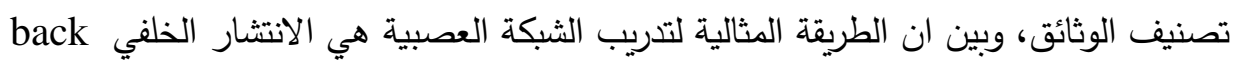
propagation الخطأ, قدمت الثبكة العصبية نتائج جيدة إلا أن تدريب الشبكة يكون بطيئًا [35]. 5. في عام 2002 قدم الباحثان Zuben و Decastro بحثاً يتناول التصنيف باستخدام خوارزمية الانتقاء النسيلي،

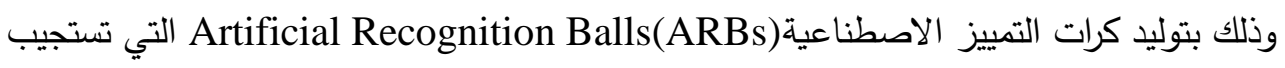
للمستضدات (مجموعة متجهات التدريب)، الكرات الأكثر استجابة تُخزن في مخزن خلايا الذاكرة وتُكون أداة التصنيف، استخدمت الطفرة في الخوارزمية مما جعل ARBs غير مماثلة لمتجهات التدريب من جهة، ومن جهة أخرى تكون متثابهة كفاية لتدريب متجهات أخرى, بيّن البحث قدرة نظام المناعة على التعلم وكفاءته في عندي

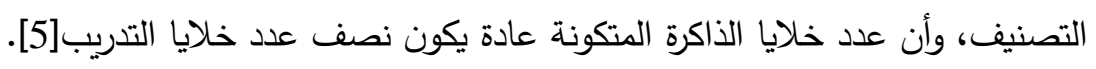
6. في عام 2005 قدم الباحثون Paab و Nurnberger و بحثاً عن استخدام مصنف المجاور الأقرب

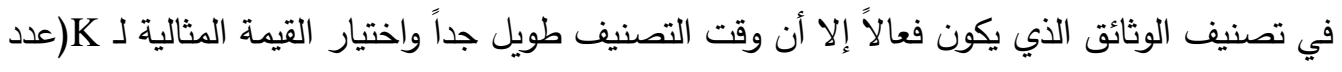
المجاورات) تعد قضية صعبة؛ إذ إن اختيار قيمة واحدة لـ K تلائم كل التطبيقات أمراً مستحيلاً [14]. 7. في عام 2007 قدم الباحثان Romero و منريقة لاستخلاص الكلمات ذات الدلالة من الوثائق النصية بالاعتماد على نظام المناعة؛ إذ تعتمد على خلفية رياضية التي تُعرف بطريقة قياسية: التفاعل بين الأجسام

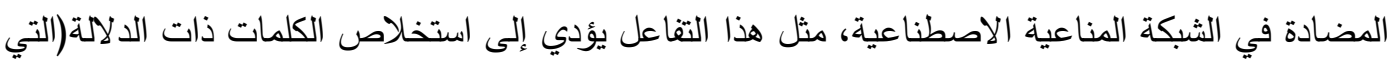

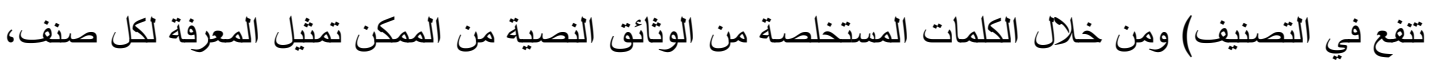

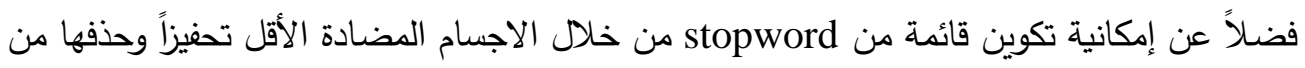
الشبكة(حذف الكلمات ذات التحفيز القليل من الثبكة)(34]. 
8. في عام 2008 قدم الباحث Tan بحثاً يتناول استخدام طريقة Centroid لتصنيف الوثائق الذي يعد من الطرائق الإرشادية الثائعة في التصنيف والمعروف ببساطته, قدم هذا الباحث تحسيناً على المصنف بإضافة

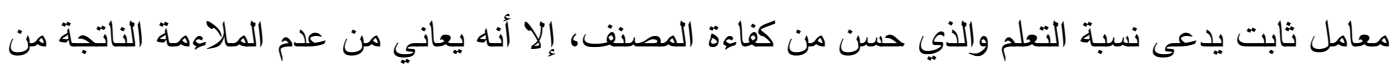

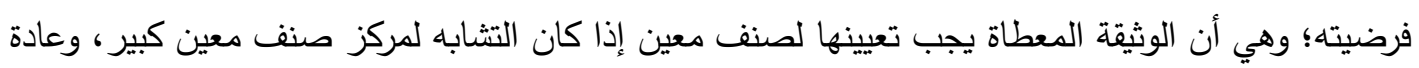
يكون هذا الافتراض غير صحيح في الحالات العملية[37].

9. في عام 2012 قدم الباحثان • Pawar و Gawande بحثا يقدم مقارنة بين انواع مختلفة لطرق التصنيف: Naïve Bayes ، المجاور الاقرب، اشجار القرار ,الثبكات العصبية ,خوارزمية Rocchio وآلة آلة دعم المتجه Support Vector Machine (SVM) اشجار القرار , فضلا عن تقنيات اختيار الخواص ودورها في زيادة دقة المصنف. 10. في عام 2015 قدم الباحث Onan بحثا يتناول النظام المناعي الاصطناعي وفاعليته في تصنيف صفحات الويب واستخدام خوارزمية immunes_1 و immunes99, ثم مقارنتهما بتقنيتي التعلم C4.5 مصنف فئف شجرة

Naïve Bayes القرار و مصنف وسن مورد

ومن خلال التجارب اثبت ان الانظمة المناعية الاصطناعية تحقق اداءاً افضل لتصنيف صفحات الويب. 11. في عام 2015 قدم الباحثان Saranya و Thenmozhi بحثا يتناول تمثيل معنى النص لتقليل الخواص باستخدام Word Net ثم تطبيق الة دعم المتجه Support Vector Machine (SVM) لتصنيف الوثائق

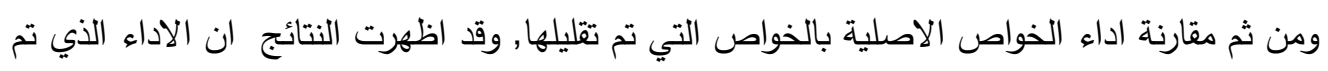
الحصول عليه بواسطة البيانات المقللة افضل من البيانات الاصلية.

12. في عام 2017 قدم الباحثان Semberecki و Maciejewski بحثا عن استخدام الثبكة العصبية مع وحدات (Long Short Term Memory)(LSTM), واختبار عدد من طرق تمثيل متجه الخواص ومن خلال التجارب اثبت ان النهج القائم على شبكة (LSTM) مع الوثائق المثثلة بمتجه متسلسلات من الكلمات المشفرة يفوق الوثائق الممثلة بمتجه خواص تكرارات الكلمة.

3. جهاز المناعة الطبيعي

لقد تمت دراسة جهاز المناعة عند الإنسان بصورة جيدة للا يزيد عن المئة عام, ولكن لا يزال هذا الجهاز لم

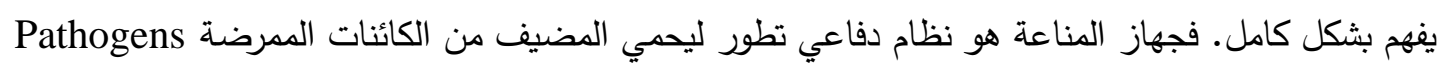

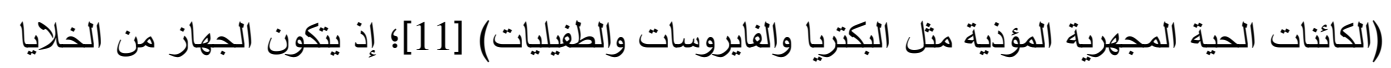
المتخصصة المتتوعة التي تتتشر وتراقب الجسم وكذلك جزيئات خلوية إضافية متتوعة وتنظيمات مناعية تعمل على توفير بيئة للخلايا المناعية لتتفاعل وتتمو وتستجيب[15]. تتشأ الخلايا المناعية في نخاع العظم والتوتة وتئة وعند نضوجها تهاجر إلى الأنسجة متنقلة عبر الأوعية الدموية واللمفاوية. إن الوظيفة الرئيسة لتلك الخلايا هي تمييز وجود العناصر الغريبة في الجسم وتعمل على الاستجابة لتتخلص منها أو لتقضي على تأثير الداخلين الأجانب[20]. تدعى المواد التي يمكن أن تحفز استجابات معينة في الجهاز

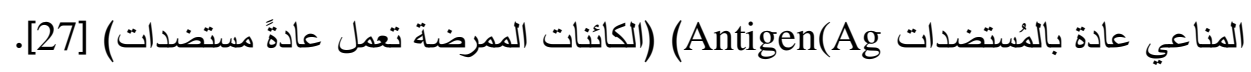


إن نظام المناعة الطبيعي يتكون من خط دفاعي ذي طبتين تعرفان بأنها جهاز المناعة الفطري وجهاز

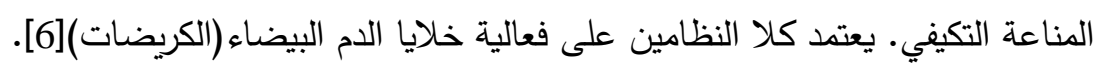

1.3 جهاز المناعة الفطري

جهاز المناعة الفطري (غير المكتسب) هو الخط الأول للدفاع الذي يعمل على تقديم استجابة فورية ولكنها

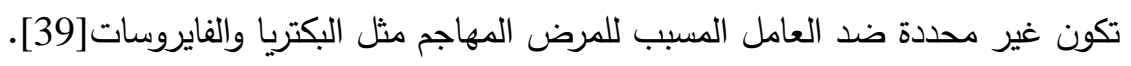

2.3 جهاز المناعة التكيفي

يثار إلى جهاز المناعة التكيفي أيضاً بأنه جهاز المناعة المكتسبة؛ إذ تطلق عليه هذه التسمية؛ لأنه مسؤول

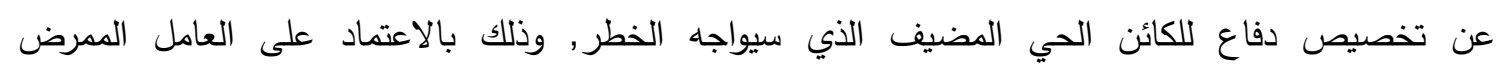
المحدد،على عكس جهاز المناعة الفطري، يوجد جهاز المناعة المكتسبة بالفقريّات فقط (الحيوانات ذات العمود الفقري)؛ إذ يحتظظ الجهاز بذاكرة للهجمات التي واجهها[2]. إن جهاز المناعة التكيفي هو خط الدفاع الثاني الذي يتوسط استجابة محددة ومتأخرة [39].

ينظم جهاز المناعة المكتسبة حول صنفين من الخلايا: الخلاياالتائية T-Cell والخلاياالبائية B-Cell، صنف خلايا جهاز المناعة الفطري أكثر تعدداً، وبضمنها الخلايا القاتلة Natural killer (NK) الخلايا التغصنية (DCs) والخلايا التَلْعَيةِ الكبيرة. تتفرع الخلايا ضمن هذه الأصناف إلى أنواع مختلفة، مثل خلية البسيطة أو المساعدة، وخلية DC غير الناضجة أو شبه الناضجة أو الناضجة [38].

NIS 4. نظريات

توجد نظريات مختلفة عن دراسة علم المناعة فيما يتعلق بالسلوك الوظيفي والتتظيمي مابين الخلايا اللمفاوية

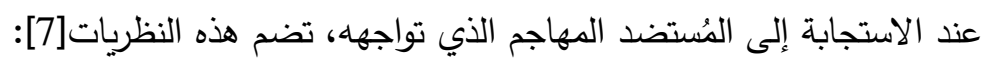
1- - 2 - النظرية التقليدية. 2- نظرية الانتقاء النَسيليّ. 3- ن نظرية شبكة المناعة. 4- نظرية الخطر.

1.4

إن النظرية التقليدية لجهاز المناعة هي إن جهاز المناعة يميز مابين ما هو طبيعي (ذاتي) وغريب (غير

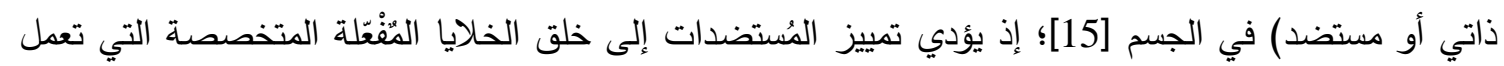
على خمول وتدمير هذه المُستضدات. يتكون جهاز المناعة الطبيعي من الخلايا اللمفاوية وأعضاء لمفاوية، هذه الأعضاء هي اللوزتان، الغدد،

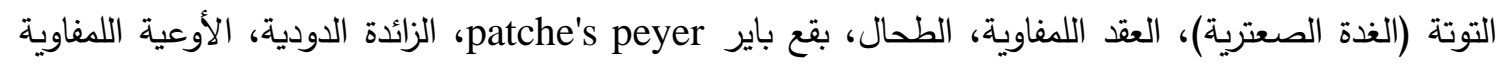

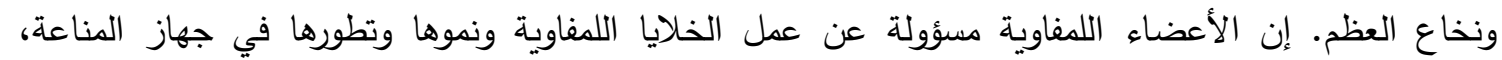
تستخدم الخلايا اللمفاوية للكشف عن أي مستضدات موجودة في الجسم، ويعمل جهاز المناعة على مبدأ نظام

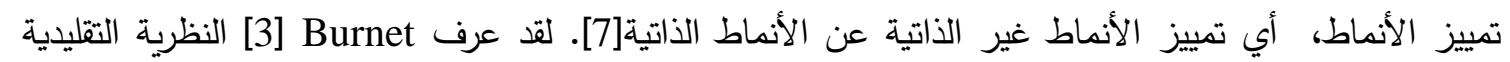


الأولى بأن الجهاز المناعي يتكون من الخلايا البائية والخلايا التائية القاتلة مع مستقبلات مستضد معين. إذ تطلق

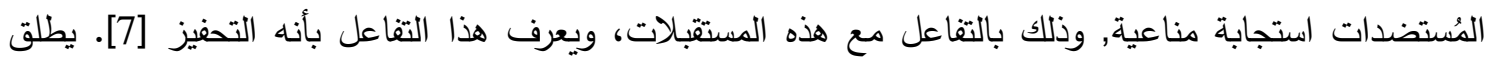

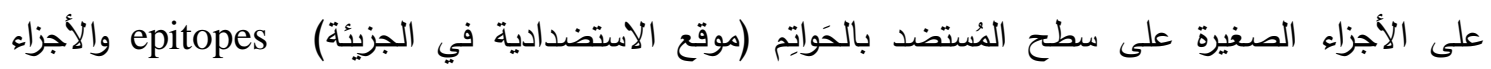

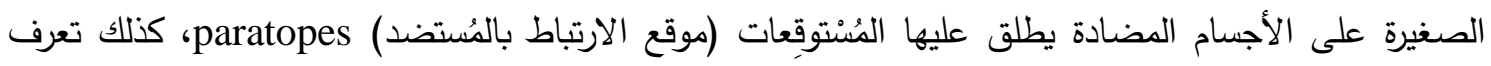

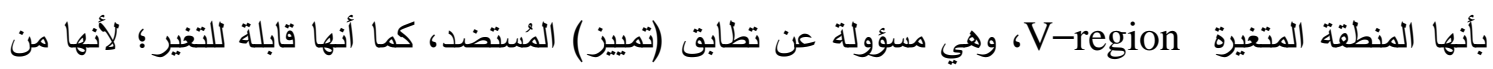

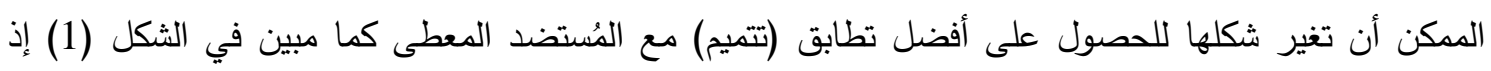

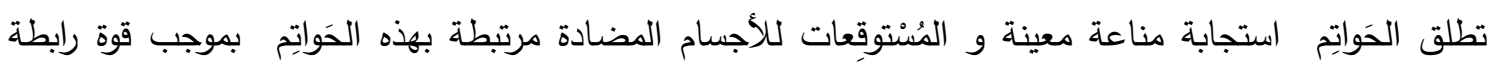

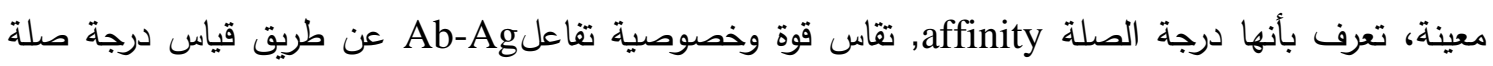

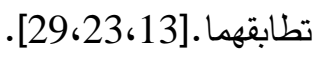

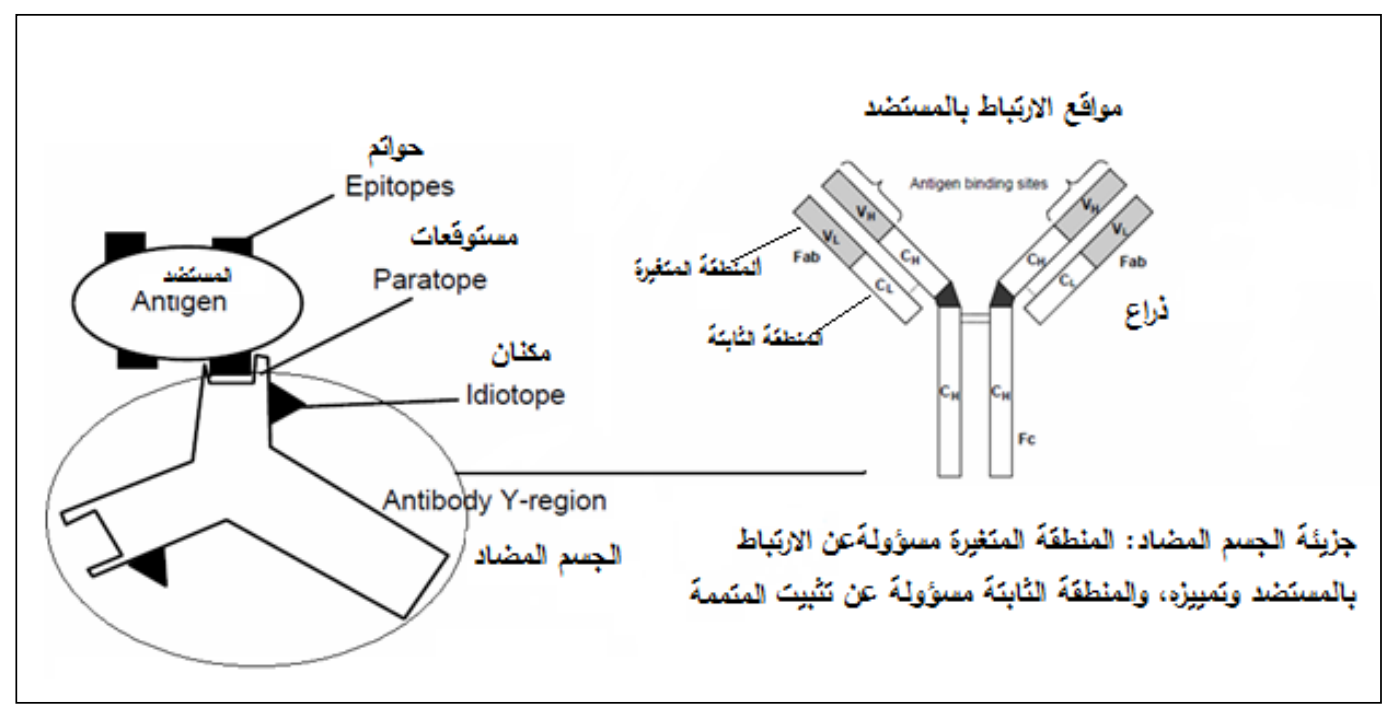

الثُكل (1) المُعقد الجسم المضادـ المُستضد[6]

إن الخلايا البائية مسؤولة عن إنتاج الأجسام المضادة وإفرازها وهي بروتينات خاصة ترتبط بالمُستضد,

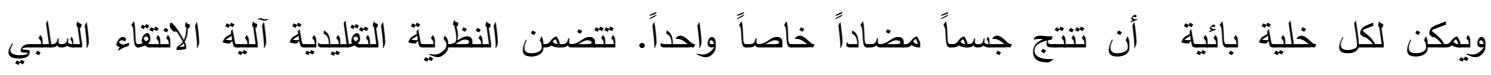
والإيجابي. 1.1.4 - م آلية الانتقاء السلبي

إن هدف الانتقاء السلبي هو تجهيز قدرة تحمل(ميزة السماحية) للخلايا الذاتية؛ إذ تتعامل مع قدرة جهاز المناعة على اكتثاف المُستضدات غير المعروفة في حين لا يتفاعل مع الخلايا الذاتية في الوقت نفسه [9]؛ وأثناء تولد

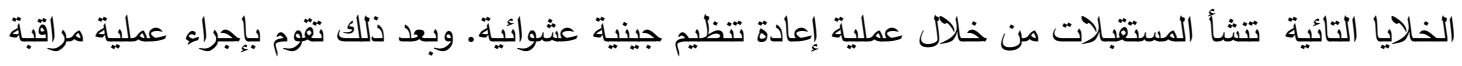

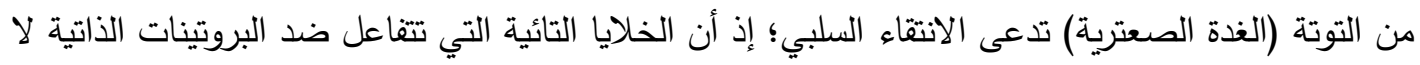

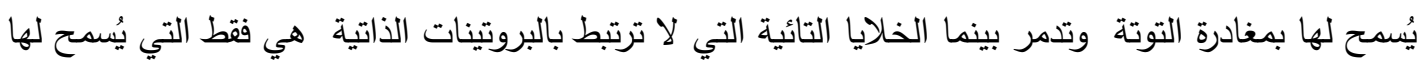

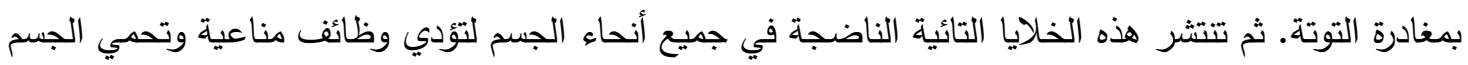

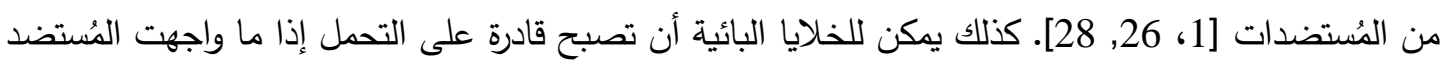
في غياب كل من الخلية التائية المساعدة والتأثيرات التحفيزية. وكما هو الحال مع الخلايا التائية، فإنه يُمكن للخلايا 
البائية المتفاعلة ذاتياً أن تفر من الانتقاء السلبي المركزي للخلية البائية, وفي هذه الحالة ستكون فعالية الخلية البائية

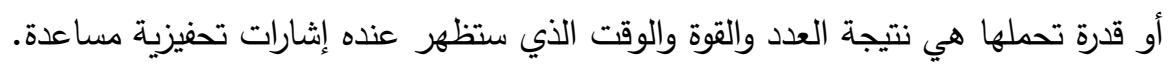

2.1.4 2. آلية الانتقاء الايجابي

يهدف الانتقاء الايجابي للخلايا اللمفاوية (البائية والتائية) إلى تجنب تجميع الخلايا اللمفاوية غير المفيدة

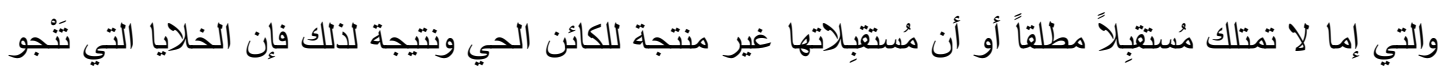

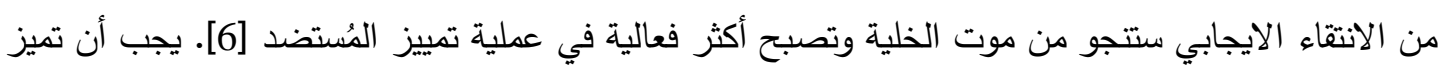

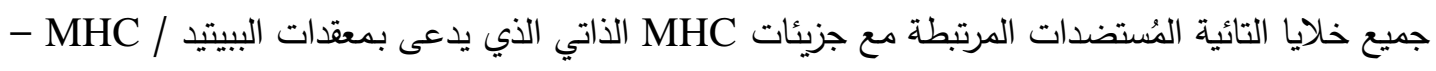

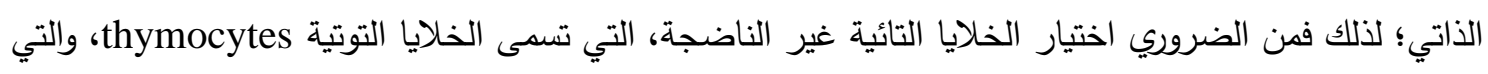
تكون مستقبلاتها قادرة على التمييز والارتباط مع جزيئات MHC - الذاتية [24]. تضمن عملية الانتقاء الايجابي

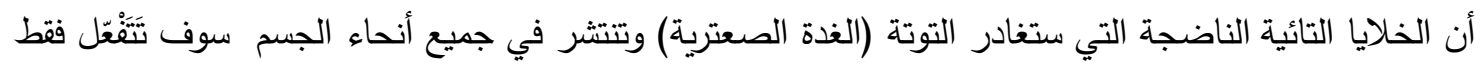
بواسطة المُستضدات الغريبة التي تقدمها جزيئات MHC - الذاتية, يتضمن أيضاً الانتقاء الايجابي الخلايا البائية الناضجة وإنقاذها من الموت. عند المقارنـة مـع الخلايا التائية، فإن الانتقاء الايجابي للخلايـا البائية الناضجة يبدو مماثلاً جداً للانتقاء

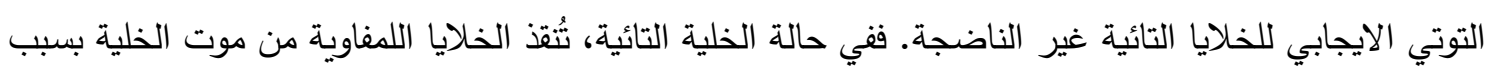

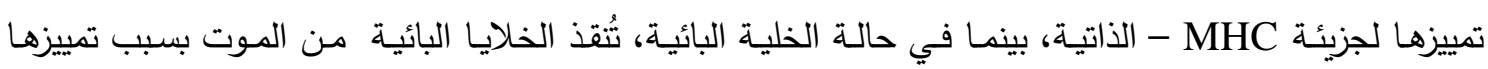
الجزيئات غير الذاتية بوجود الإشارات التحفيزية [6]. 2.4. نظرية الانتقاء النَسيليّ يصف مبدأ الانتقاء النسيليّ الصفات الأساسية لاستجابة المناعة لمحفزات المُستضد، الفكرة الأساسية لهذه

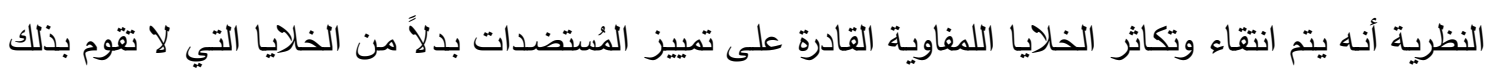

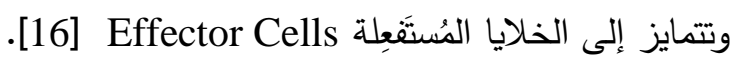

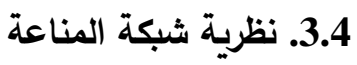

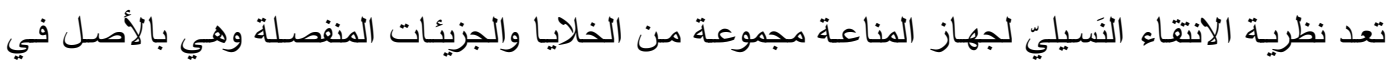

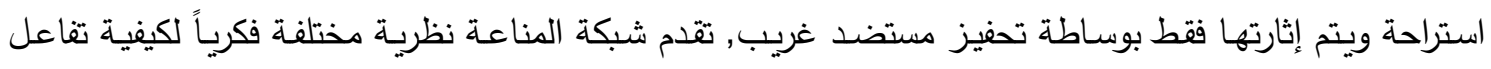

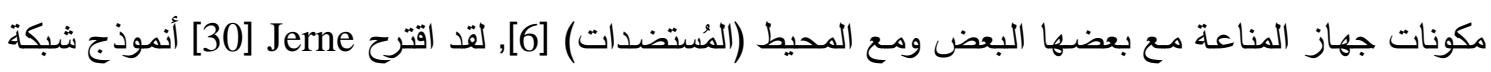

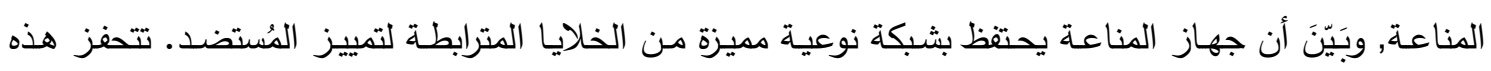
الخلايا وتثبط إحداها الأُخرى بطريقة معينة تؤدي إنى إلى استقرار الثبكة.

4.4. نظرية الخطر وابليط

قدمَت عالمة المناعة Polly Matzinger في عام 1994 نظريـة الخطر [22]. ذكرت أن جهاز المناعة

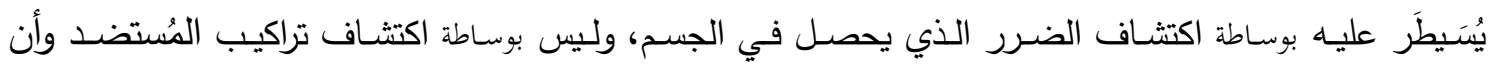

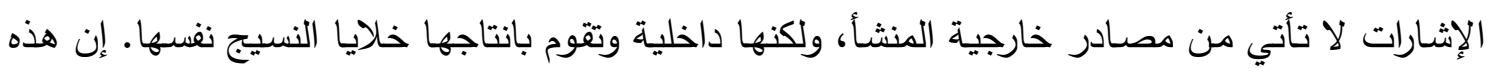
الإثـارات الداخلية تسمى إشارات الخطر danger signals [12].يوضح الثكل (2) نظرية الخطر [27], وتعمل الخلية التغصنية بمبدأ نظرية الخطر . 


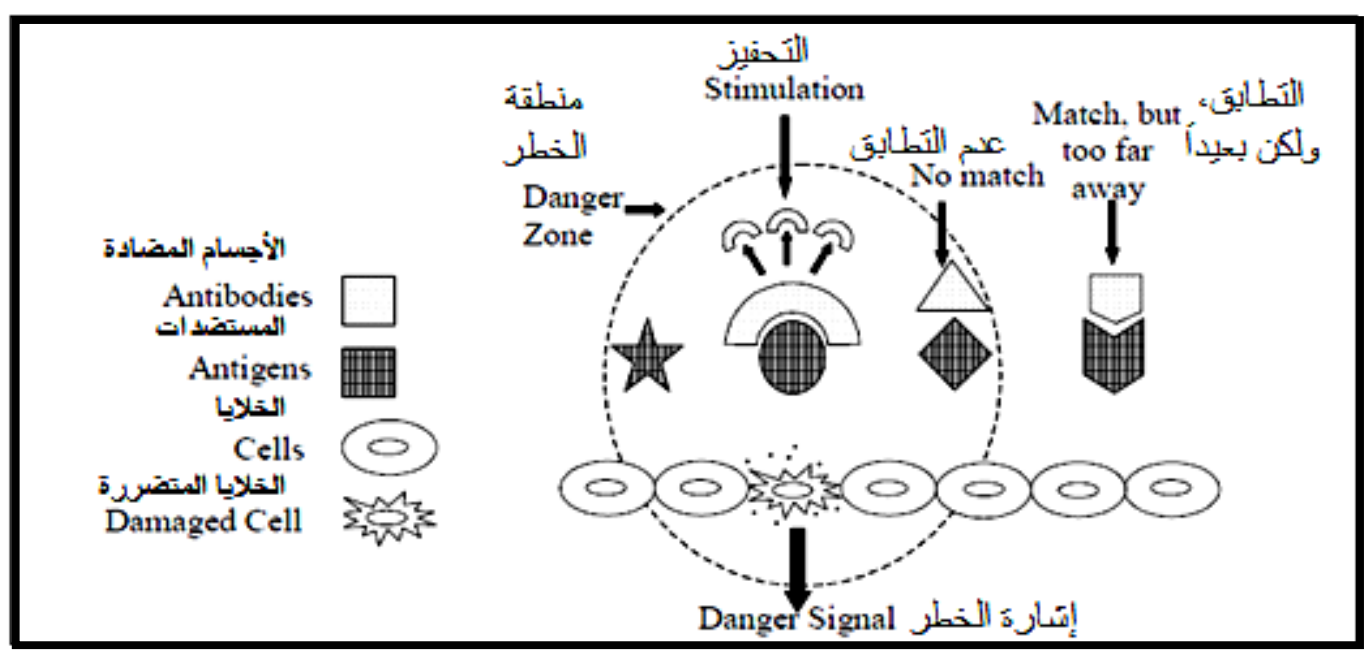

الثكل (2) أنموذج نظرية الخطر]

5. 5ظام المناعة الاصطناعي

AIS

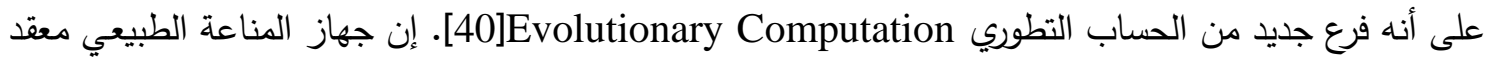
جداً ليتم مُحاكاته صناعياً، ولكن نجح- A.B. Watkins بمحاكاة أهم وظائف جهاز المناعة الطبيعي فيما يخص تمييز الأنماط. إن نظام المناعة الاصطناعي، هو مجموعة من خوارزميات التصنيف الذكية، التي تستخدم آلية دفاع المناعة الطبيعية لأغراض تقانية، قادرة على التكيف والتعلم. لذلك انتشر هذا المفهوم في تطبيقات تقانة عديدة

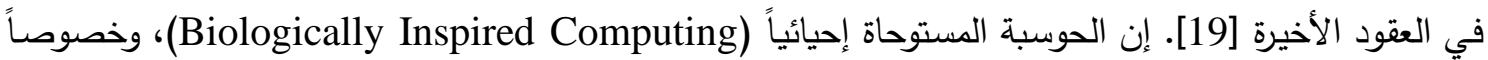
أنظمـة المناعـة الاصـطناعية (AIS) هـي حل واعد لتطـوير أدوات دفاعيـة مكيفـة ومؤتمتــة للتهديـدات الحاليـة

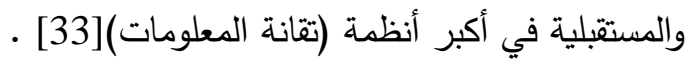
قدم De.Castro و Timmis [6] إطار عمل مستخدم بصورة أكثر شيوعاً في هندسة AIS، والموضح

في الثكل (3). (3) (2) (2)

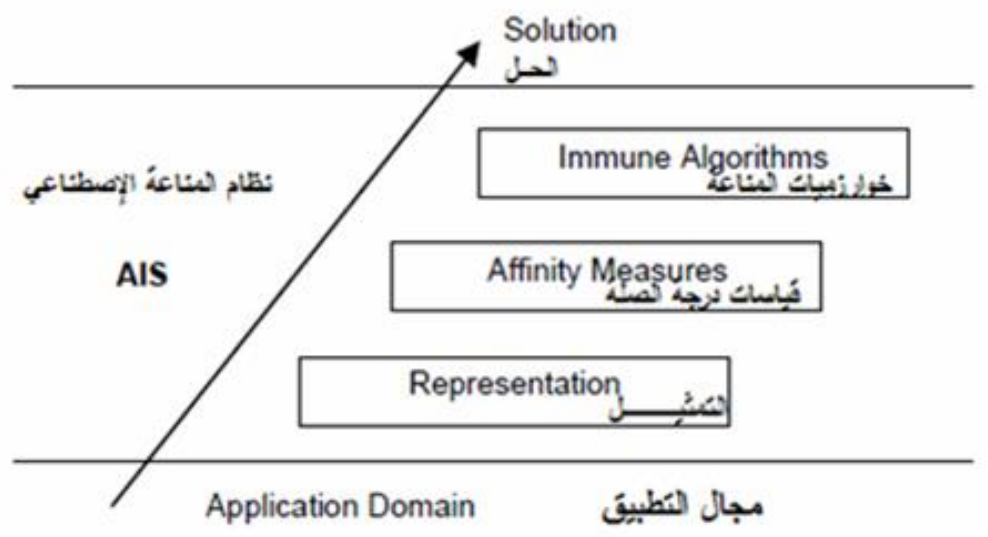

[6] AIS الشكل (3) طبقات إطار عمل

يتضمن إطار العمل ثلاث خطوات مستقلة بشكل نسبي في بناء (AIS). إن أساس كل نظام هو مجال

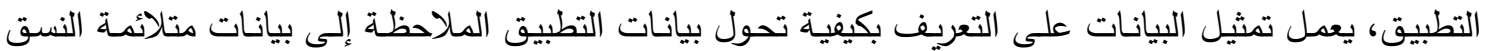


وذلك لتعالج بوساطة خوارزميات (AIS). تحتاج البيانات المستخدمة أن تكون مُنسقة لتكون قادرة على قياس درجة

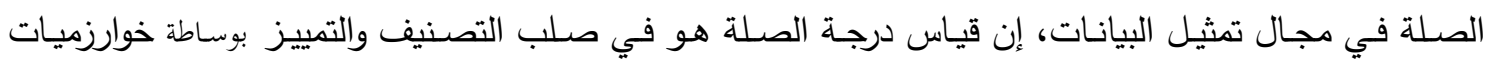

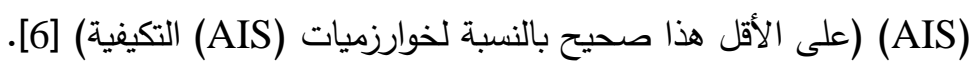

\section{6. مصنف أنموذج الانتقاء السلبي والايجابي الهجين}

طور في هذا البحث أنموذج التصنيف HPNS الذي يعتمد على نظام المناعة التكيفي؛ إذ تم في هذاء

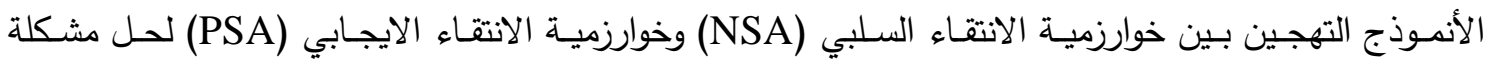

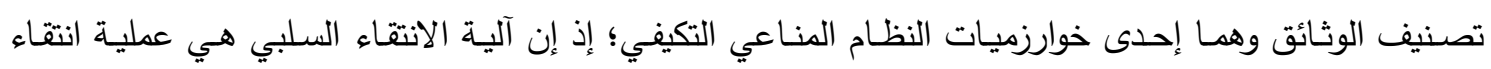

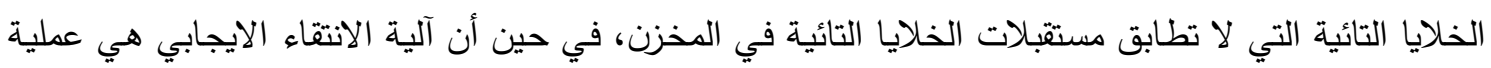

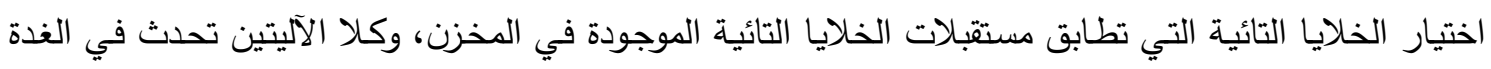
التوتية.

لقد وظفت الفكرة ذاتها؛ إذ يقوم أنموذج النظام المناعي المصنف المبني على الخوارزمية الهجينة(HPNS)،

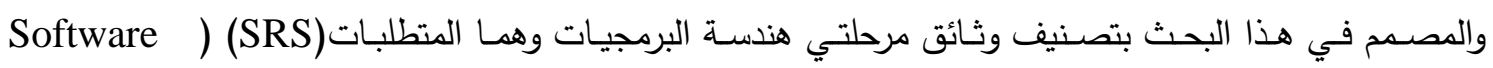
• (Software Test Document) (STD) والاختبار (Requirement Specification يوضح الجدول (1) عملية التماثل بين نظام التصنيف المناعي الاصنياعي والجهاز المناعي الطبيعي.

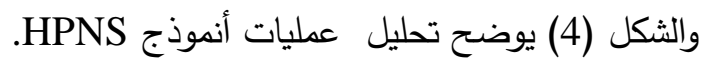
الجدول (1) التماثل بين جهاز المناعة الطبيعي و نظام التصنيف المناعي الاصطناعي

\begin{tabular}{|c|c|c|}
\hline نظام المناعة الاصطناعي & نظام المناعة الطبيعي & العناصر \\
\hline الوثائق & المستضدات & 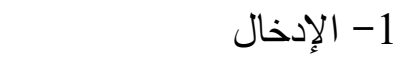 \\
\hline Stop words & الخوجودة في التيائيـة الذاتيـة (غيـر ناضــة & 2- المعلومات المخزونة \\
\hline القاموس & الخلايـا التائيـة الناضـجة الموجودة في & 3- البيانات المدربة \\
\hline نظام التصنيف & الغدة التوتية & 4- البيئة \\
\hline ناتج نظام التصنيف & استجابة نظام المناعي الطبيعي & 5- 5 أسلوب الرد \\
\hline متجه الخواص & البيتيدات & 6- جزء من بيانات الإدخال \\
\hline
\end{tabular}

1.6. وصف أنموذج الانتقاء السلبي والايجابي الهجين

تتكون خوارزمية التصنيف التكيفي (HPNS) من ثلاث وحدات أساسية:

$$
\begin{aligned}
& \text { 1- - وحدة المعالجة الأولية. } \\
& \text { 2- وحدة التدريب . } \\
& \text { 3- وحدة الاختبار. }
\end{aligned}
$$


تتفاعل هذه الوحدات مع بعضها البعض لانجاز مهمة تصنيف الوثائق والثكل (5) يوضـح عمل الوحدات بصورة

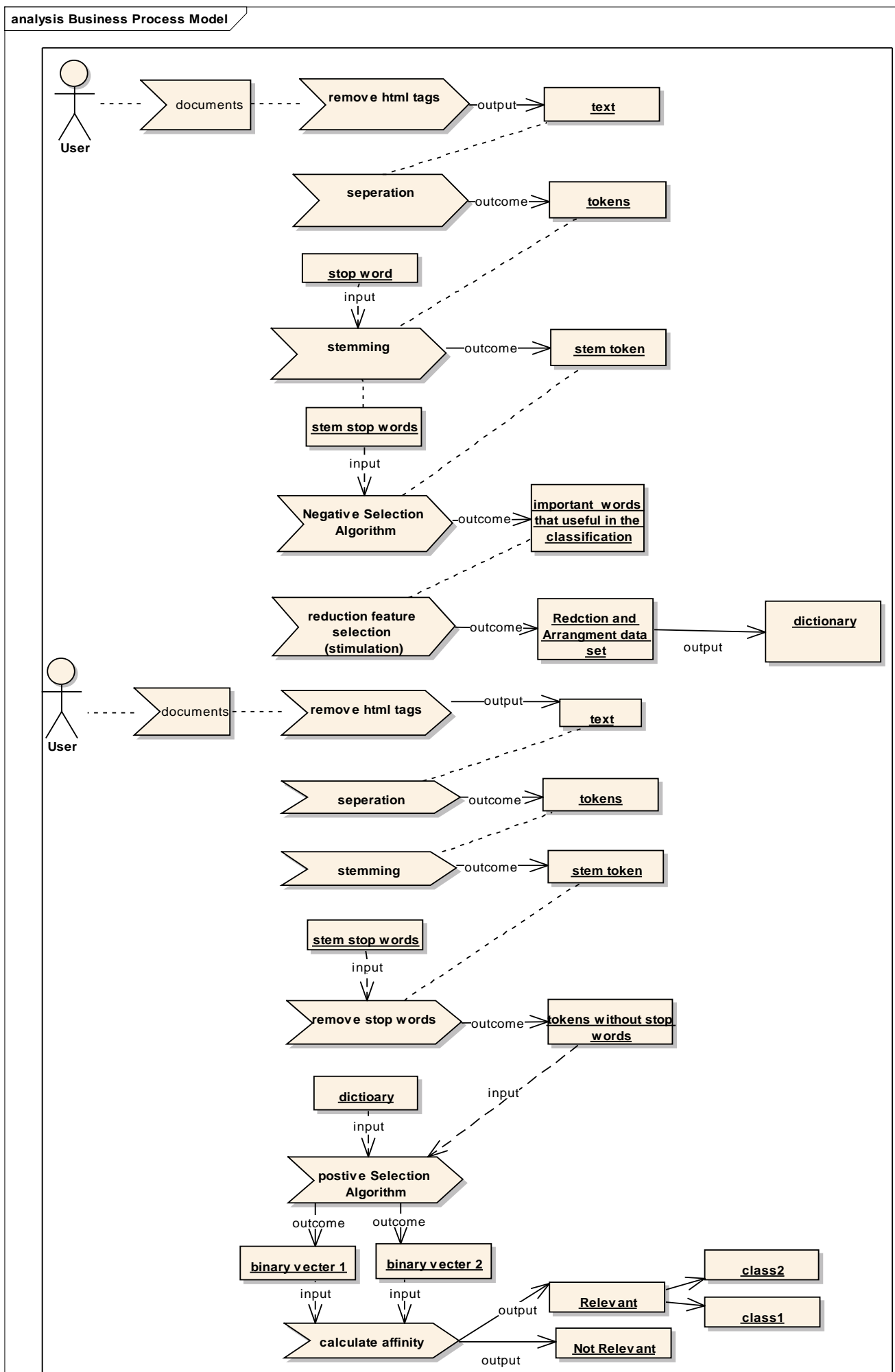

HPNS الثكل(4) أنموذج عمليات 


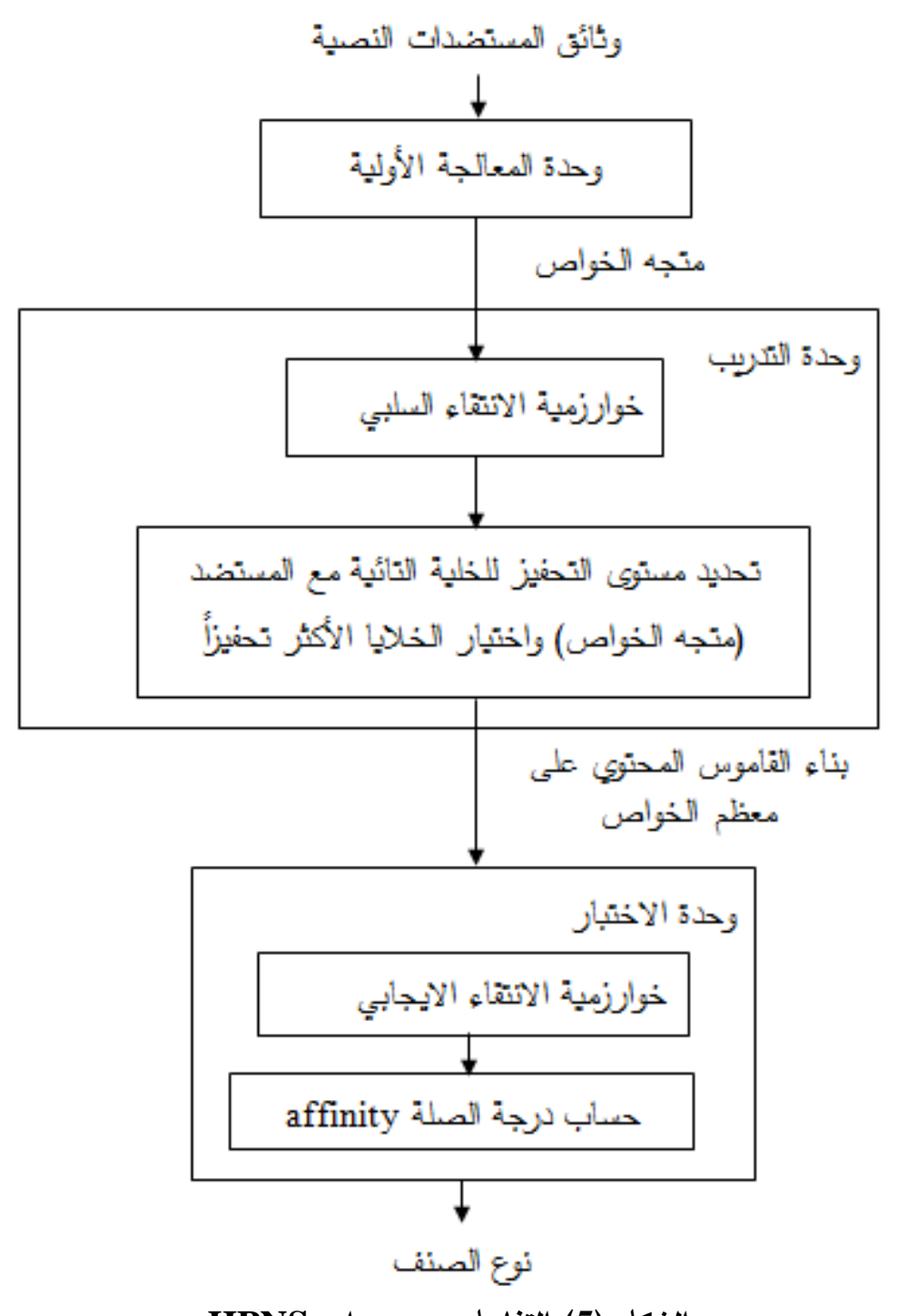

الثكل (5) التفاعل بين وحدات HPNS

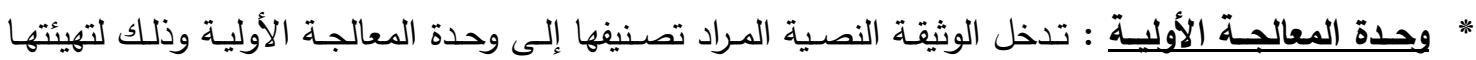
وتحويلها إلى صيغة يسهل على وحدة التدريب التعامل معها, إن معالجة الوثيقة النصية هي خطوة مهمة جداً

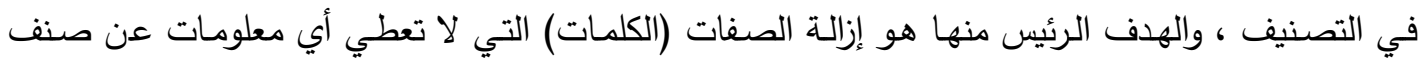
الوثيقة, فضـلاً عن إزالة البيانات المتكررة, تتضمن عملية المعالجة استخلاص الخواص عن طريق تحويل التهل وثيقة النص المدخلة إلى متجه خواص الصفات، ويضم التحويل الخطوات التالية:

1- تحويل الوثيقة من نوع (HTML) إلى نص واضـح (Jlain Text) وذلك بإزالة الــ(Java Script)

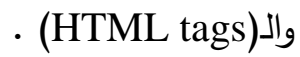
2- إزالة الأرقام وعلامات التتقيط وتقسيم النص إلى كلمات منفصلة. 3- ازالة التكرار وتكوين قائمة بكلمات الوثيقة.

4- تحويل الكلمات الموجودة في القائعة إلى أحرف كبيرة ( Upper Case Letters)

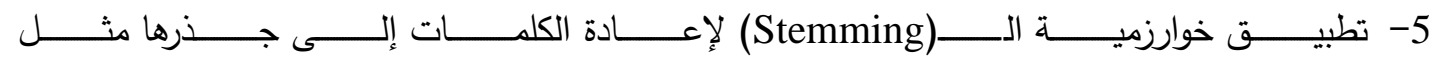
Testing, Tested, Testes $\rightarrow$ Test 
* وحدة التدريب : تتألف من مرحلتين: المرحلة الأولى : استخدام خوارزمية الانتقاء السلبي (NSA) لتوليد القاموس (الكاشف) لكل صنف.

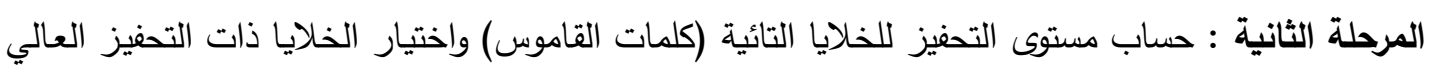

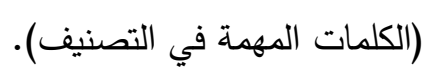

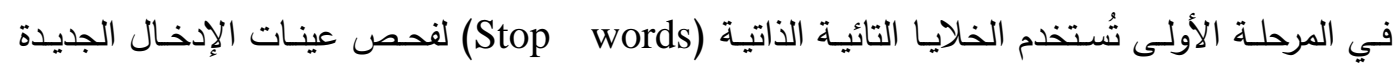

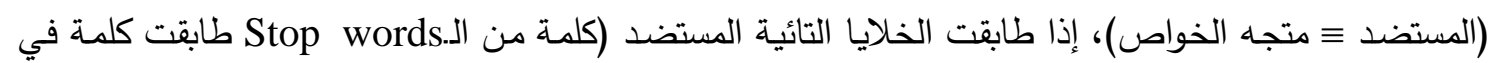

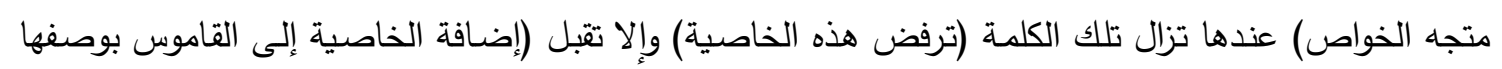

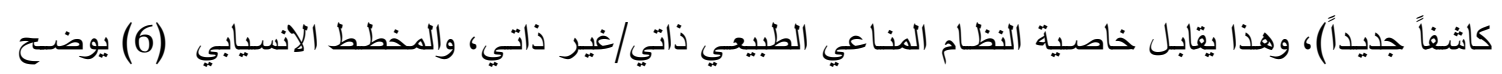

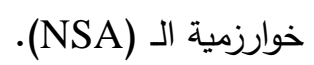
وفي المرحلة الثانية حُسب مستوى التحفيز للخلايا التائية (كلمات القاموس) واختيار الخلايا ذات التحفيز العالي (الكلمات المهمة في التصنيف) باستخدام طريقة (Mutual Information (MI) كما مبين في المعادلة الفيانة رقم)(1) $M I(t, c)=\log \frac{P(c, t)}{P(t) \cdot P(c)}$

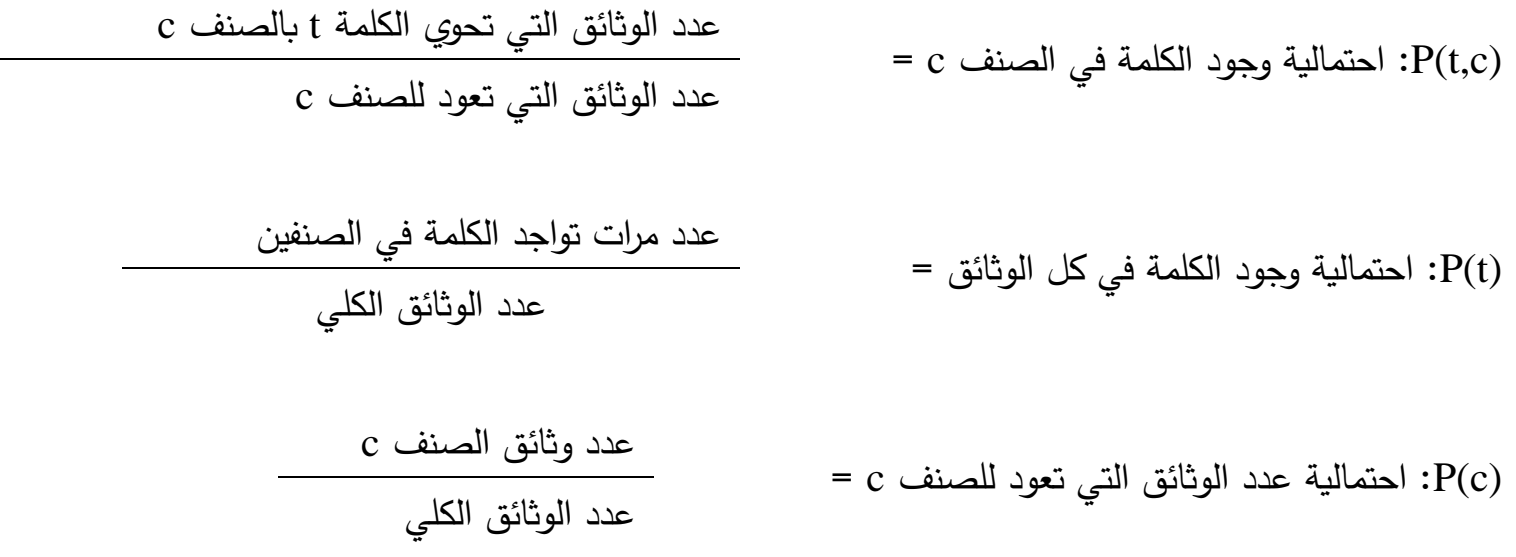

بعد تطبيق طريقة (MI) على كواشف القاموس أصبح لكل كاشف (كلمـة) قيمة تحفيز خاصـة بها,

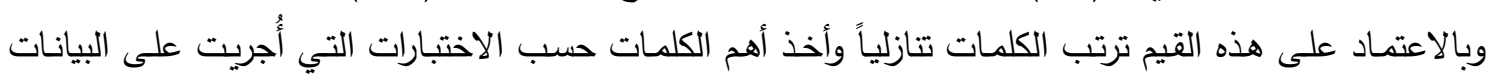

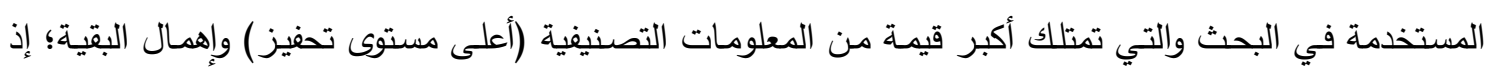

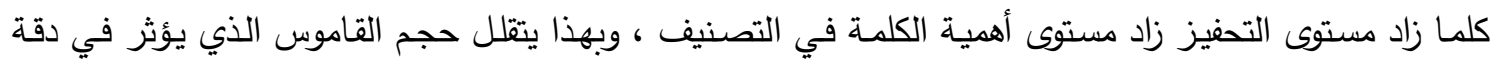

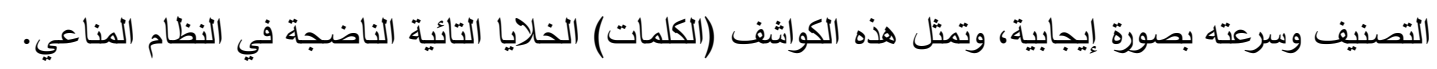




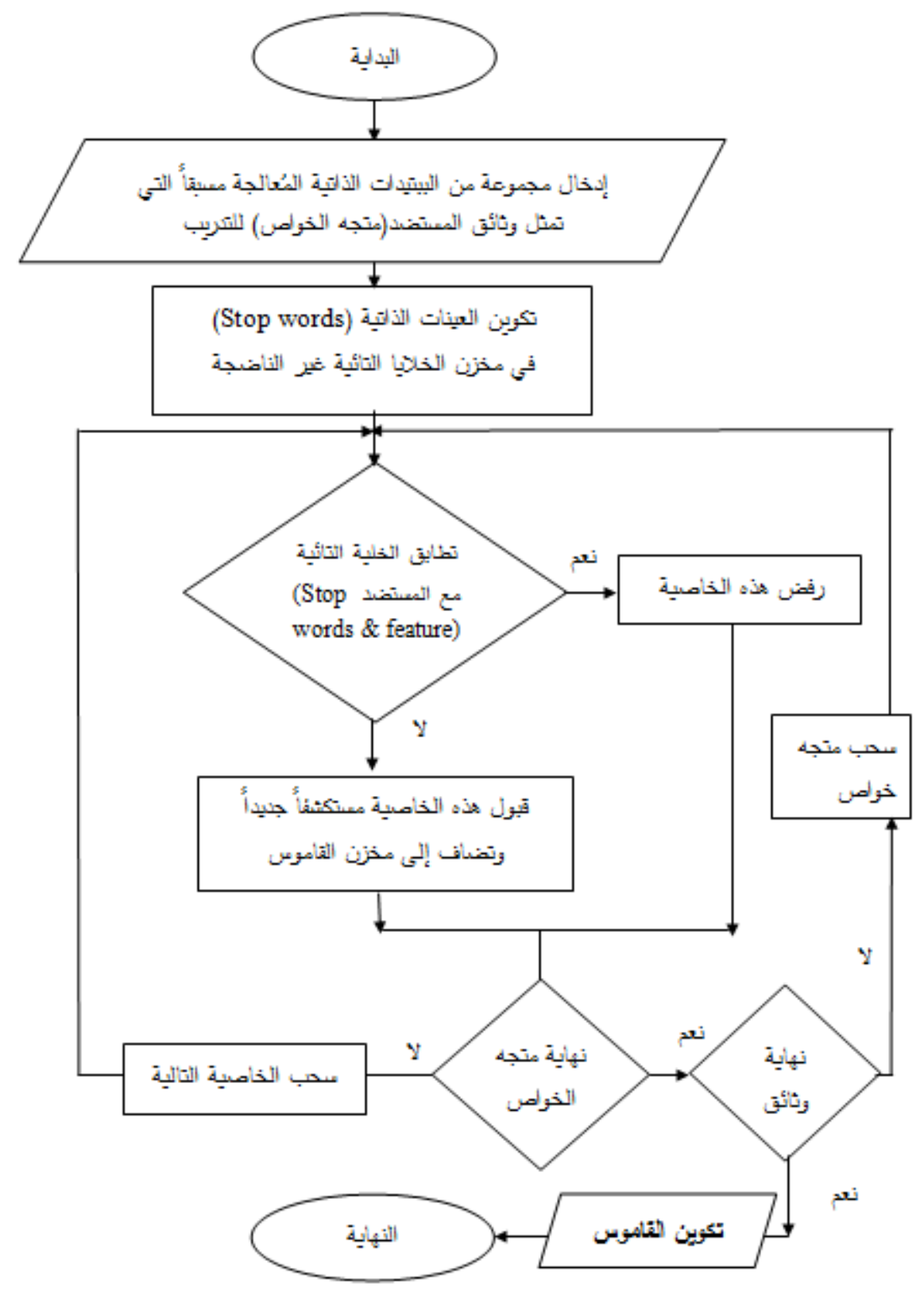

الثكل (6) المخطط الانسيابي لخوارزمية الـ(NSA)

بعـد تطبيق طريقـة (MI) على كواشـف القـاموس أصبح لكل كاشـف (كلمـة) قيمسة تحفيز خاصـة بهـا وبالاعتماد على هذه القيم ترتب الكلمات تتازلياً وتؤخذ أهم الكلمات حسب الاختبارات التي أُجريت على البعل البيانات

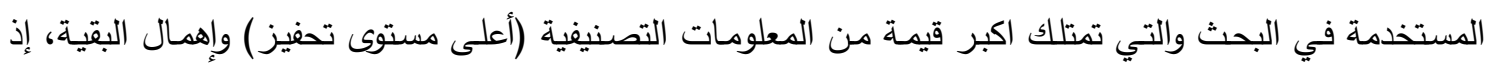

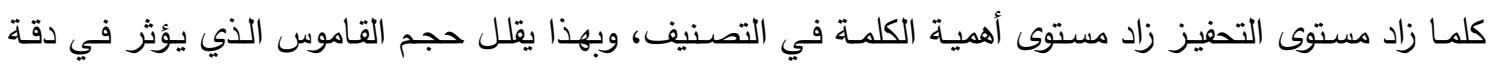
التصنيف وسرعته بصورة إيجابية، وتمثل هذه الكواشف (الكلمات) الخلايا التائية الناضجة في النظام المناعي.

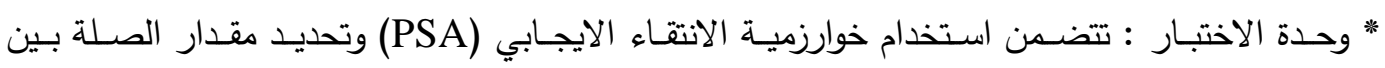
المستضد(وثائق الاختبار) والخلايا التائية لتحديد الصنف الذي تعود إليه الوثيقة, ويعمل الانتقاء الايجابي على الإنى تحفيز الخلايا التائية الناضجة القادرة على تمييز المستضد. إذا لم تميز الخلية التائية مستضداً واحداً على الأقل يتم 
حذفها وإلا يتم إختيارها بوصفها خلايا مؤهلة مناعياً Immune Competent Cell ثم حساب قيمة ترابط المستضد مع الخلايا التائية والمخطط الانسيابي(7) يوضح خوارزمية الانتقاء الايجابي مع حساب قيمة التطابق.

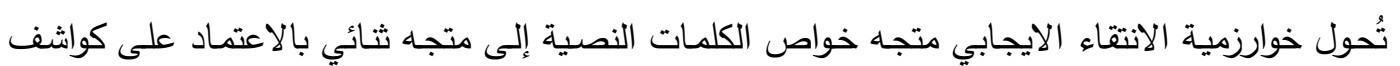

القاموس، فإن وجدت الخاصية في القاموس تمثل بالقيمة (1) في المتجه الثائي وإذا لم توجد يتم تمثيلها بـ(0).

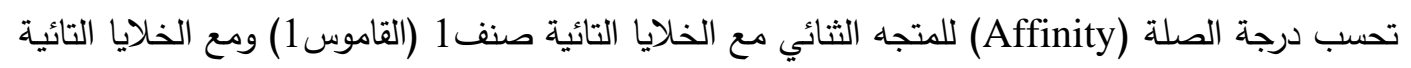
صنف2 (القاموس2) إذ إن المتجها ذا الصلة الأعلى تعني أن الوثيقة تعود لذلك الصنف، وذلك باستخدام قانون البتات المستمرة المتعددة:

قانون البتات المستمرة المتعددة = عدد الواحدات الكلي + 2عدد الواحدات المستمرة ........(2) بعد إختيار الخوارزمية المناسبة لكل وحدة وتمثيل العناصر يتم البدء بتحديد تفاصيل التصميم؛ إذ يتكون الأنموذج الأول للصنف من ثلاثة حزم (Package) :

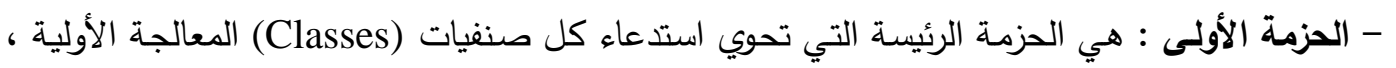

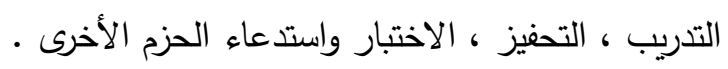
- الحزمة الثانية : هي حزمة IRutilities التي تحوي خوارزمية Porter الخاصـة بعملية (Stemming) - الحزمة الثالثة : هي حزمة Jsoup التي تقوم بتحويل وثيقة html إلى سلسلة (String) • والثكل (8) يوضـح حزم أندوذج HPNS المستخدمة في تصنيف الوثائق والصنفيات (Classes) داخل كل حزمة . بعدها تحدد المتغيرات والدوال الخاصـة بكل صنف (Class) باستخدام مخطط الصنفيات الموضـح في الأشكال (9)، (10)، (11) (19) 


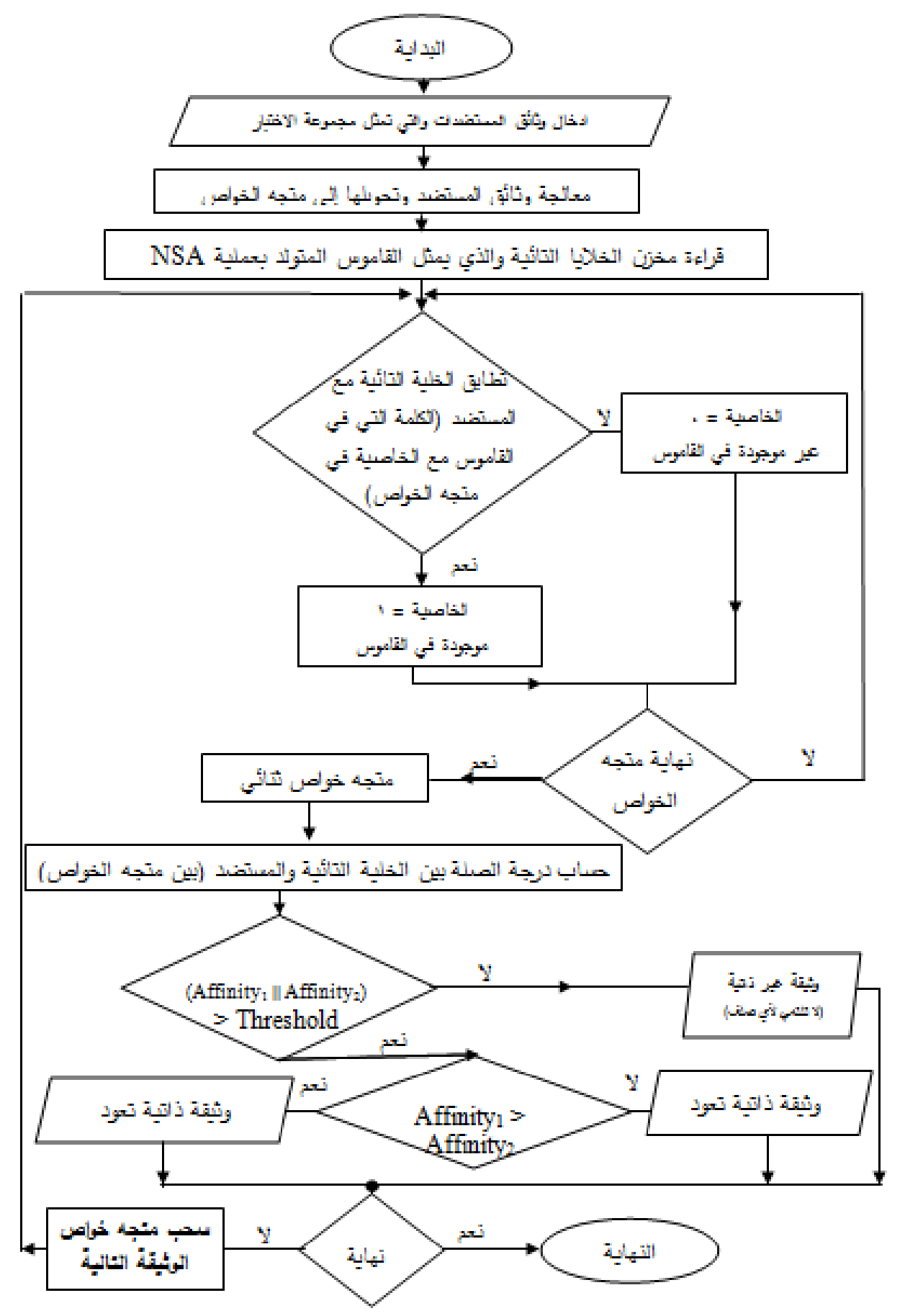

الثكل (7) المخطط الانسيابي لخوارزمية (PSA) وحساب مقار صلة المستضد والخلية التائية 


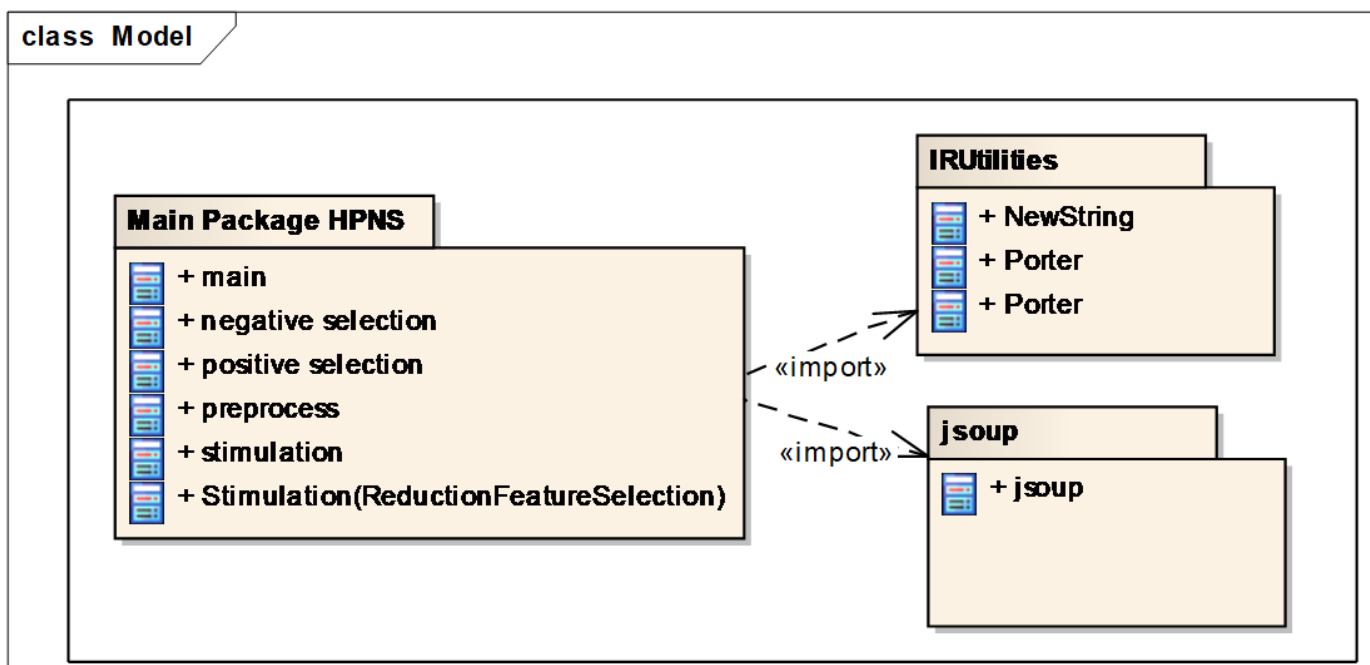

\section{الشكل (8) مخطط الصنفيات للحزم الثلاثة لأنموذج HPNS}

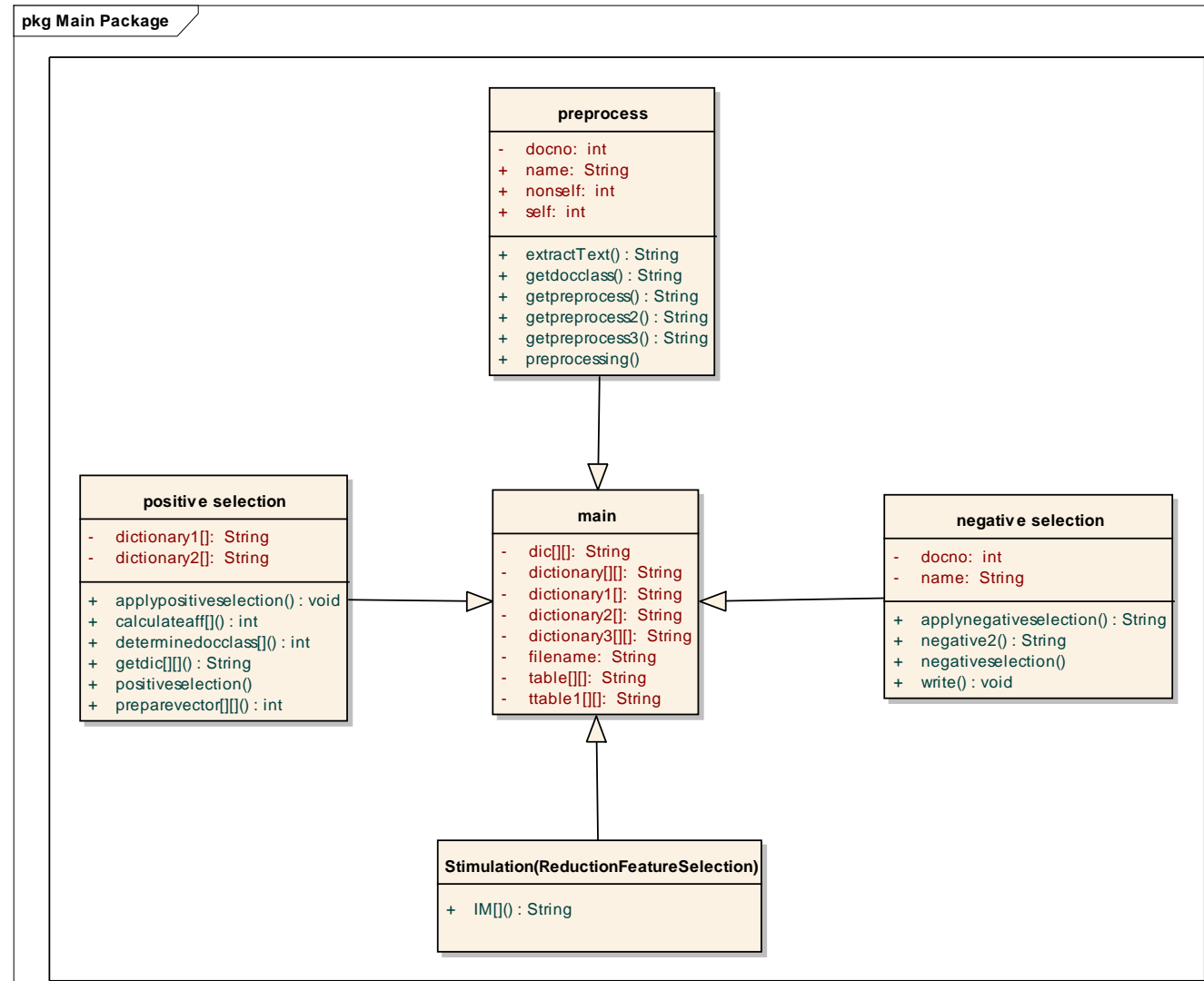

الثكل (9) مخطط صنفيات الحزمة الرئيسة لأنموذج HPNS 

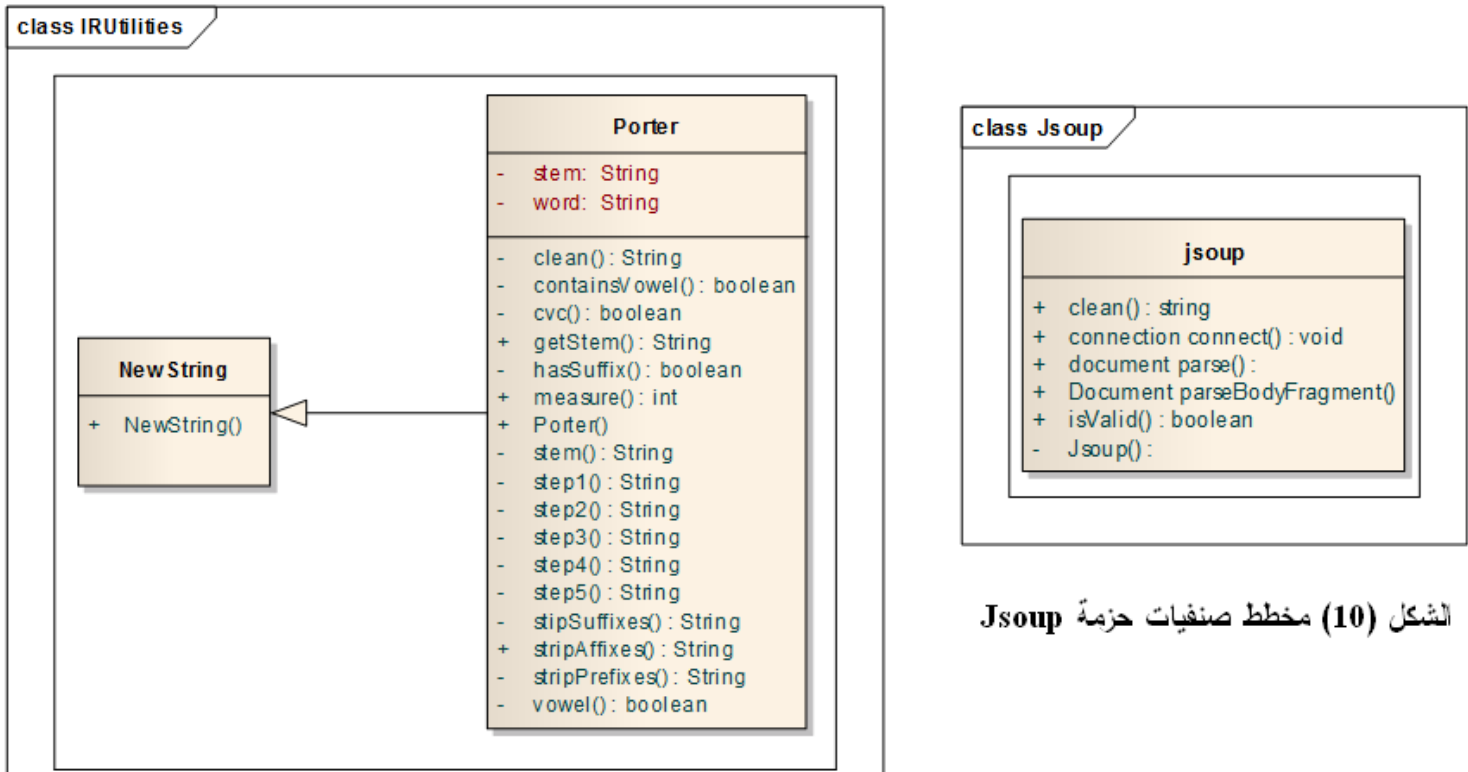

Jsoup الثنكل (10) مخطط صنفيات حزمة

IRUtilities الثكل (11) مخطط صنفيات حزمة

ثم يحـد الترتيـب الزمنـي للصـنفيات حسب التسلسـل الزمني لاستـدعائهم باسـخدام المخطط التسلسـي الموضح في الثكل (12) م sd sequence diagram

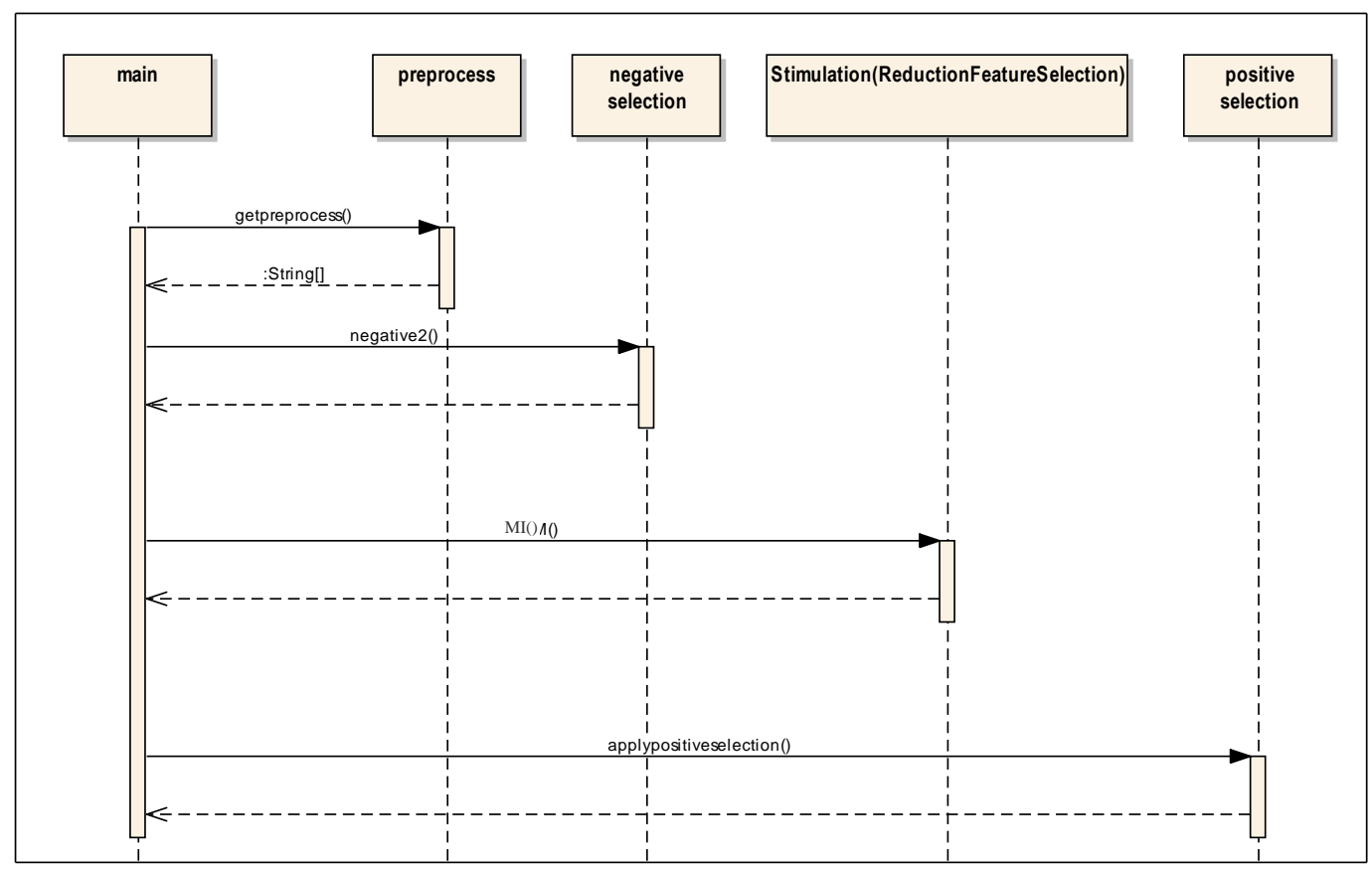

\section{الشكل (12) المخطط التسلسلي لأنموذج HPNS}

* مرحلة بناء HPNS : تضم هذه المرحلة كتابة البرنامج ويتم فيها تحويل الخوارزميات والمخططات المحددة في لادول

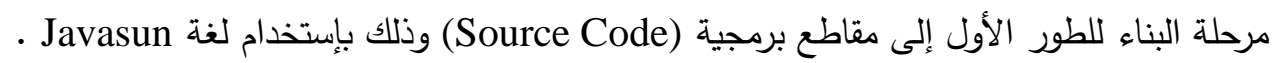




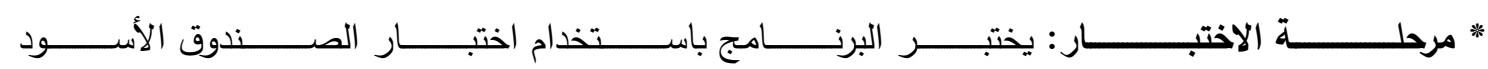
(Black Box Test)

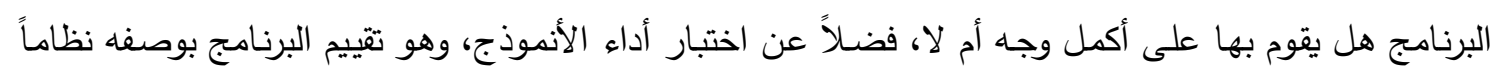
يقوم بوظائف محددة حسب المتطلبات ويندرج هذا الاختبار باختبار الوحدات الثثلاثة :

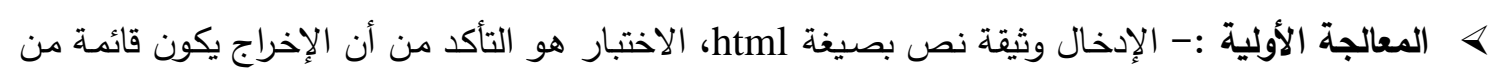

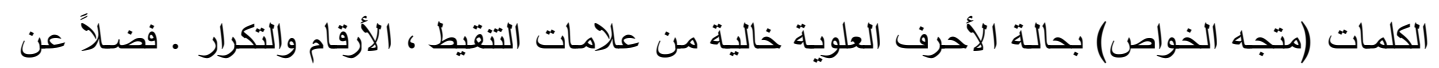

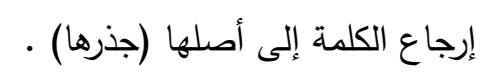

ه التدريب : الإدخال قائمة الكلمات، الاختبار هو التأكد من بناء قاموس خالٍ من الكلمات التي ليس لها أهمية

$$
\text { في التصنيف (Stopwords) وتحوي فقط الكلمات ذات الأهمية الأكبر • }
$$

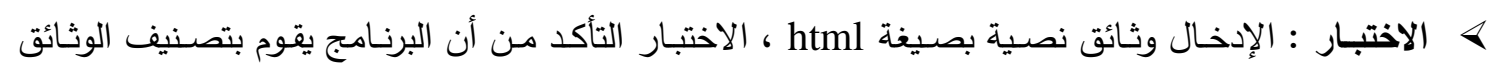
لصنفها الصحيح بالاعتماد على درجة الصلة بينها وبين القاموس المكون في مرحلة التدريب، وتقييم البرنامج.

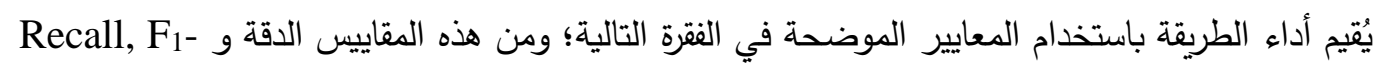

.(Tp, Tn, Fn, Fp) macro, F1-micro, Specificity وفي حالة كثف أي خطأ في البرنامج يتم الرجوع للخطوة السابقة ومعالجة المشكلة ثم التأكد مرة اخرى من صحته.

\section{* مقاييس التقييم}

هنالك طرائق عديدة لتحديد الفعالية إلا أن المقاييس الأكثر استخداما هي (precision ، recall ،accuracy).

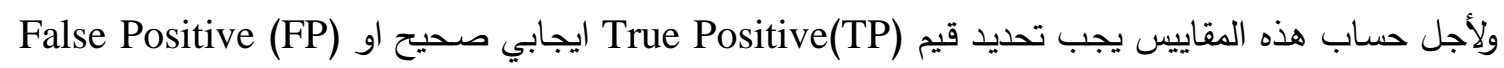
ايجابي خاطئ أو True Negative (TN) سلبي صحيح أو False Negative (FN) سلبي خاطئ الموضح في الجدول (2) (60]).

جدول (2) تصنيف الوثائق [60]

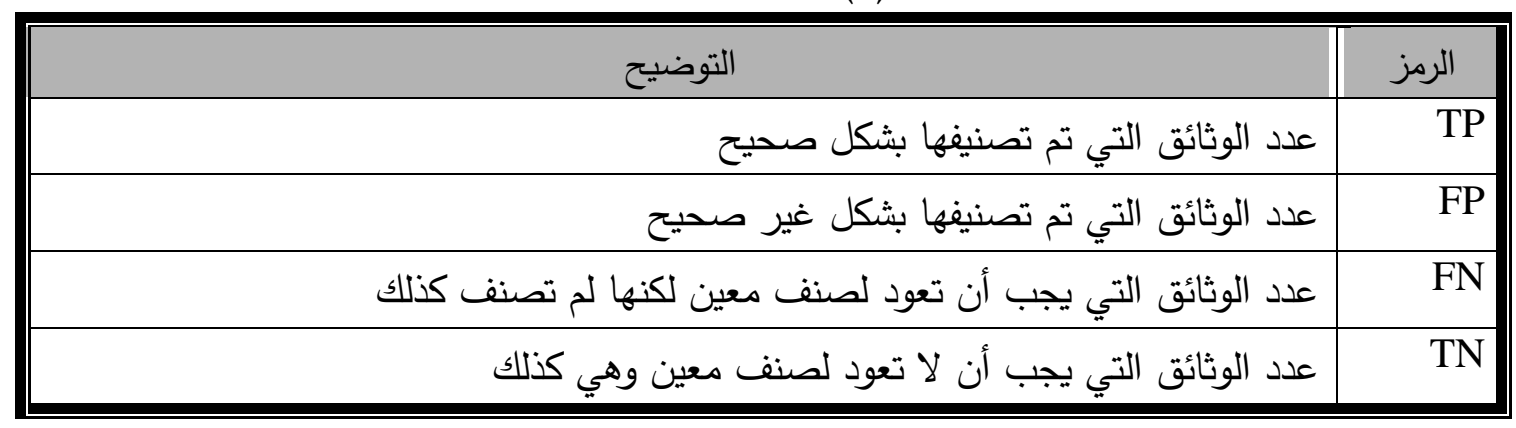

: عدد الوثائق التي تم تصنيفها بشكل صحيح. TP

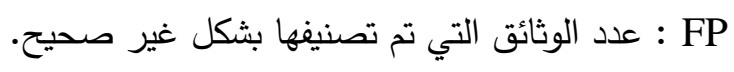

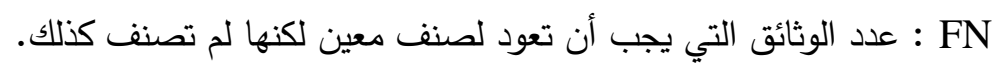

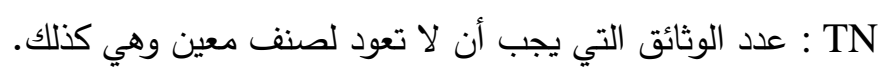




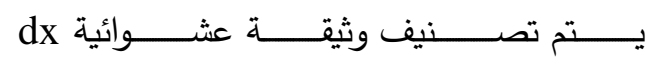
ع Precision وفقاً لـ ci أو ما يمكن ان يعد صنفاً صحيحاً.

$\pi_{i}=\frac{T p_{i}}{T p_{i}+F P_{i}}$

Recall : تعرف على انها النسبة التي يتم فيها اتخاذ قرار اذا كان يجب تصنيف وثيقة عشوائية dx تحت صنف $P_{i}=\frac{T p_{i}}{T p_{i}+F N_{i}} \ldots \ldots$

Accuracy : غالباً مـا يستخدم على اعتبار انها مقياس لأساليب التصنيف الا ان قيم Accuracy اكثر تقبلاً

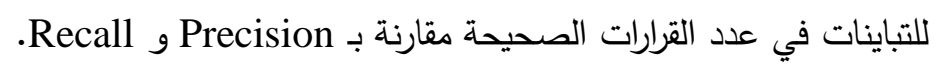
$A_{i}=\frac{T p_{i}+T N_{i}}{T p_{i}+T N_{i}+F p_{i}+F N_{i}}$

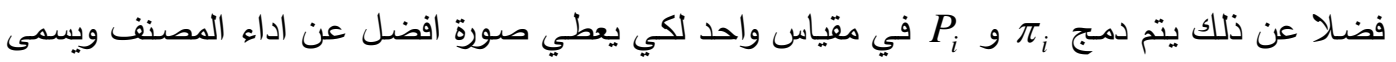

مقياس F1 والذي يتم حسابه بطريقتين Micro-average و (6) يقوم بحساب قرار التصنيف بشكل عام للصنفين كما في المعادلات : Micro-average F1-measure -1 الى (8)

$\mathrm{F} 1($ micro-averaged $)=\frac{2 \pi p}{\pi+p} \ldots$

$\pi=\frac{\mathrm{TP}}{\mathrm{TP}+\mathrm{FP}}=\frac{\sum_{\mathrm{i}=1}^{\mathrm{M}} \mathrm{TP}_{\mathrm{i}}}{\sum_{\mathrm{i}=1}^{\mathrm{M}}\left(\mathrm{TP}_{\mathrm{i}}+\mathrm{FP}_{\mathrm{i}}\right)}$

$\mathrm{P}=\frac{\mathrm{TP}}{\mathrm{TP}+\mathrm{FN}}=\frac{\sum_{\mathrm{i}=1}^{\mathrm{M}} \mathrm{TP}_{\mathrm{i}}}{\sum_{\mathrm{i}=1}^{\mathrm{M}}\left(\mathrm{TP}_{\mathrm{i}}+\mathrm{FN}_{\mathrm{i}}\right)}$

يقوم بحساب قرار التصنيف بالنسبة لكل صنف ثم يتم اخذ المعدل لهما

$$
F 1_{i}=\frac{2 \pi_{i} P_{i}}{\pi_{i}+P_{i}}
$$

$F 1($ macro - averaged $)=\frac{\sum_{i=1}^{M} F 1_{i}}{M}$.... كما في المعادلات(9) الى (10). 


\section{6. تهيئة وتنفيذ أنموذج Initialization \& Implementation of HPNS Model HPNS}

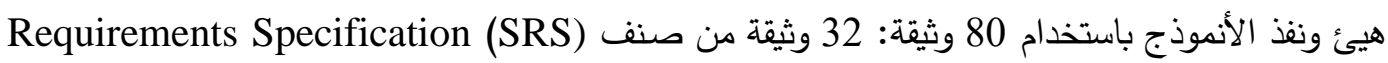

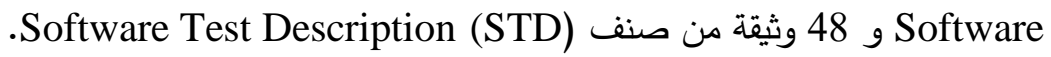

يوجد صيخ عديدة لخزن الوثائق ومن أشهرها (HTML)، وعند تهيئة النظام باستخدام هذه الوثائق تمرر بعدة مراحل لتحويلها إلى صيغة يسهل على المصنف التعامل معها. والعديد من الخواص المهمة يتم الحصول عليها خلال مرحلة التهيئة، والخطوة الأساسية في هذه المرحلة هي تحديد الخواص التي ستستخدم في مرحلة

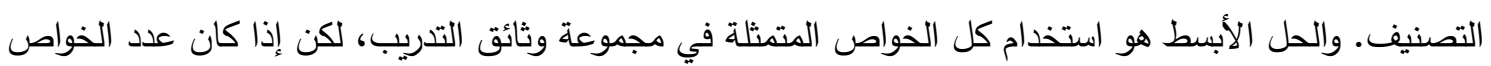
كبيراً جداً يقلل باستخدام تقانات مختلفة بخطوات متعددة وهي إزالة كلمات stop word، إعادة الكلمات إلى جذرها stemming واختيار الخواص التي تمتلك أعلى معدل من المعلوماتية MI التي تثيد في التصنيف وخزنها في القاموس، والثكل(13) يوضح تأثير كل من هذه التقانات على تقليل عدد الخواص. تُل

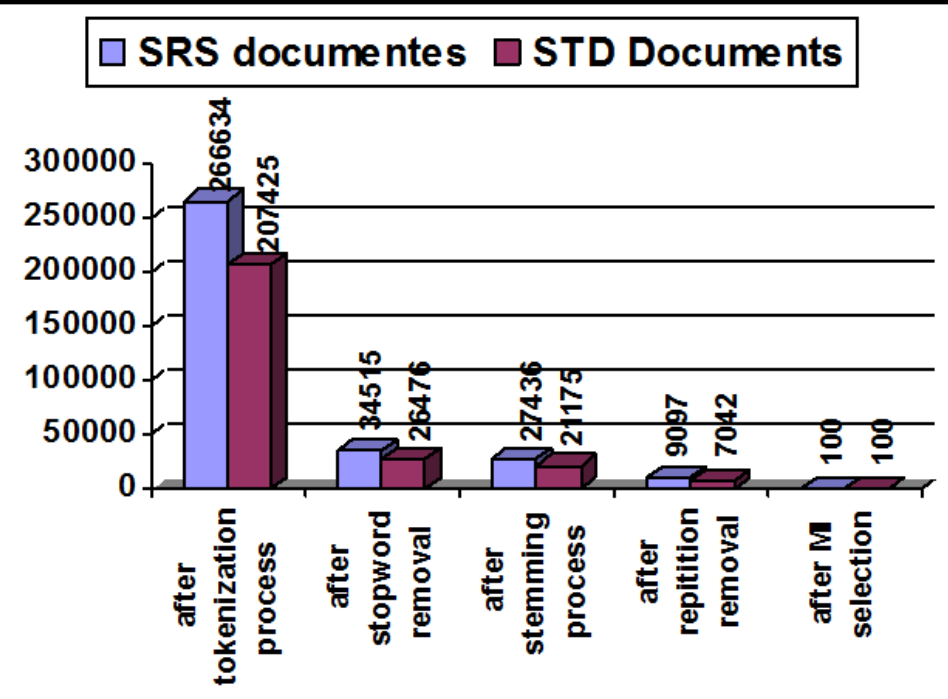

الثكل (13) تأثير العمنيات المختلفة في حجم متجه الخواص

Testing of Model and Results 3.6. اختبار الأنموذج ونتائجه اختبر الأنموذج HPNS بإدخال مجموعة من وثائق مراحل هندسة البرمجيات التي حملت من المواقع الالكترونية الخاصة بوثائق هندة البرمجيات، وتصنف الوثائق المختارة إلى واحد من الأصناف التالية :

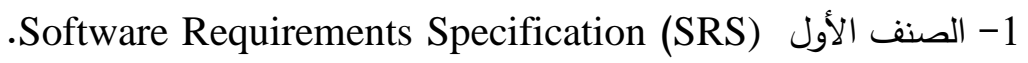

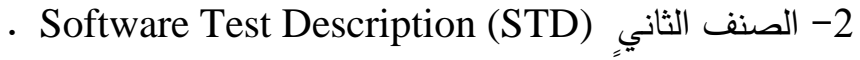
3- 3 - لا تعود للصنفين. أجري الاختبار على مجاميع باعداد مختلفة من الوثائق، الجداول والأشكال التالية توضح نتائج اختبار تصنيف وثائق هندسة البرمجيات. يوضح الجدول (3) والثكل(14) نتائج دقة تصنيف وثائق التدريب من وثائق NB و HPNS لمصنفات SWE 
الجدول(3) نتائج تصنيف وثائق التدريب (800) وثيقة

\begin{tabular}{|l|l|l|l|l|l|l|}
\hline $\begin{array}{l}\text { Features } \\
\text { Mode }\end{array}$ & 250 & $\mathbf{5 0 0}$ & $\mathbf{7 5 0}$ & $\mathbf{1 0 0 0}$ & $\mathbf{1 5 0 0}$ & $\mathbf{2 0 0 0}$ \\
\hline HPNS & $\% 100$ & $\% 97.5$ & $\% 98.75$ & $\% 96.25$ & $\% 95.0$ & $\% 93.75$ \\
\hline NB & $\mathbf{9 6 . 2 5}$ & $\mathbf{9 6 . 2 5}$ & $\mathbf{9 3 . 7 5}$ & $\mathbf{9 3 . 7 5}$ & $\mathbf{9 0 . 0}$ & $\mathbf{9 1 . 2 5}$ \\
\hline
\end{tabular}

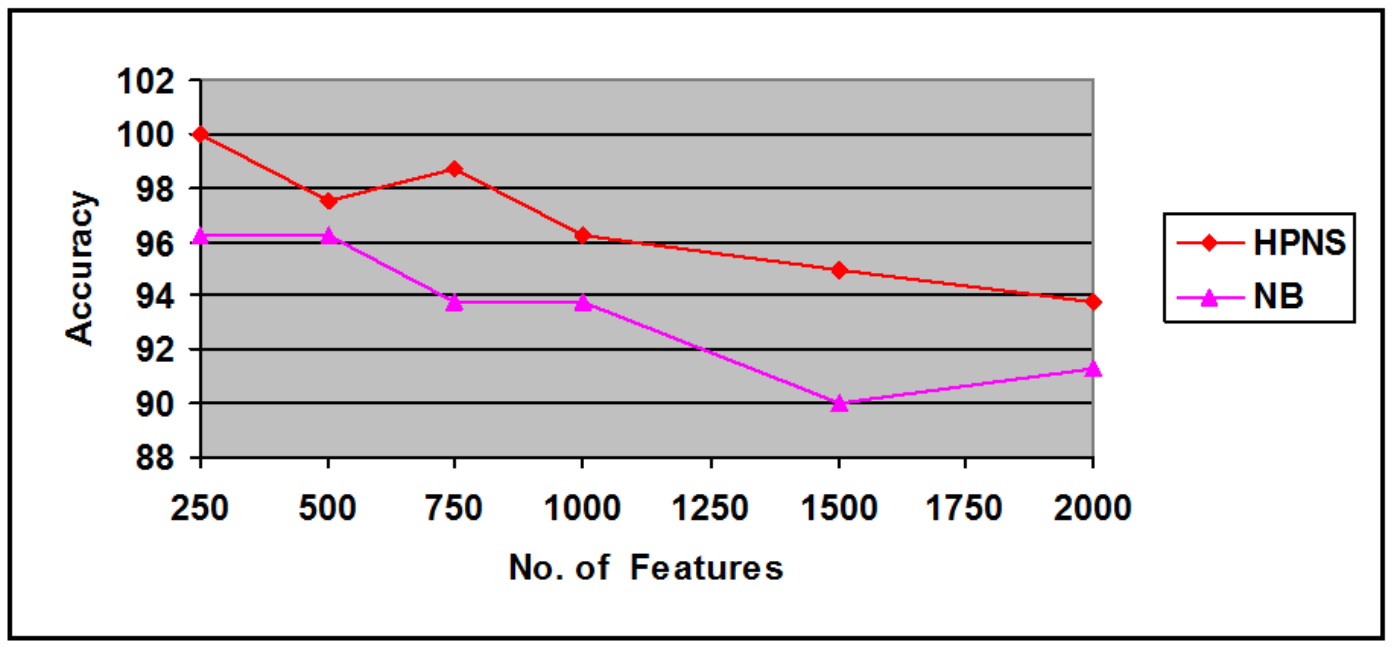

الثنكل(14) دقة المصنفات نوثائق التثريب

يوضتح الجدول (4) والثكل(15) نتائج دقة تصنيف (40) وثيقة اختبار من وثائق SWE للمصنفات

.NB و HPNS

الجدول(4) دقة تصنيف (40) وثيقة اختبار

\begin{tabular}{|c|c|c|c|c|c|c|}
\hline Model Features & 250 & 500 & 750 & 1000 & 1500 & 2000 \\
\hline HPNS & $\% 95$ & $\% 95$ & $\% 95$ & $\% 92.5$ & $\% 92.5$ & $\% 92.5$ \\
\hline NB & 90 & 87.5 & 87.5 & 87.5 & 87.5 & 90 \\
\hline
\end{tabular}

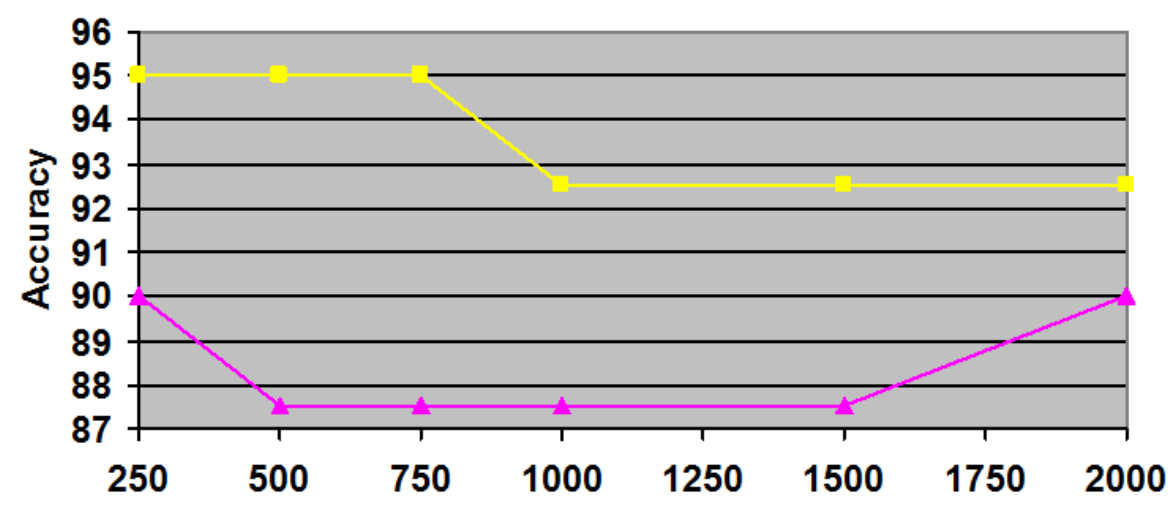

No. of Features

الثنكل(15) دقة المصنفات نوثائق الاختبار 
يوضح الجدول (5) والثكل(16) نتائج دقة التصنيف لمجاميع وثائق التدريب والاختبار الكلي

والبالغ (120) وثيقة.

الجدول(5) دقة تصنيف الوثائق الكلية (التدريب والاختبار) (120) وثيقة

\begin{tabular}{|l|l|l|l|l|l|l|}
\hline $\begin{array}{l}\text { Features } \\
\text { Model }\end{array}$ & 250 & 500 & 750 & 1000 & 1500 & 2000 \\
\hline HPNS & $\% 96.67$ & $\% 95.83$ & $\% 96.67$ & $\% 94.17$ & $\% 93.33$ & $\% 92.5$ \\
\hline NB & 94.17 & 95.0 & 92.5 & 90.0 & 87.5 & $\mathbf{9 0 . 0}$ \\
\hline
\end{tabular}

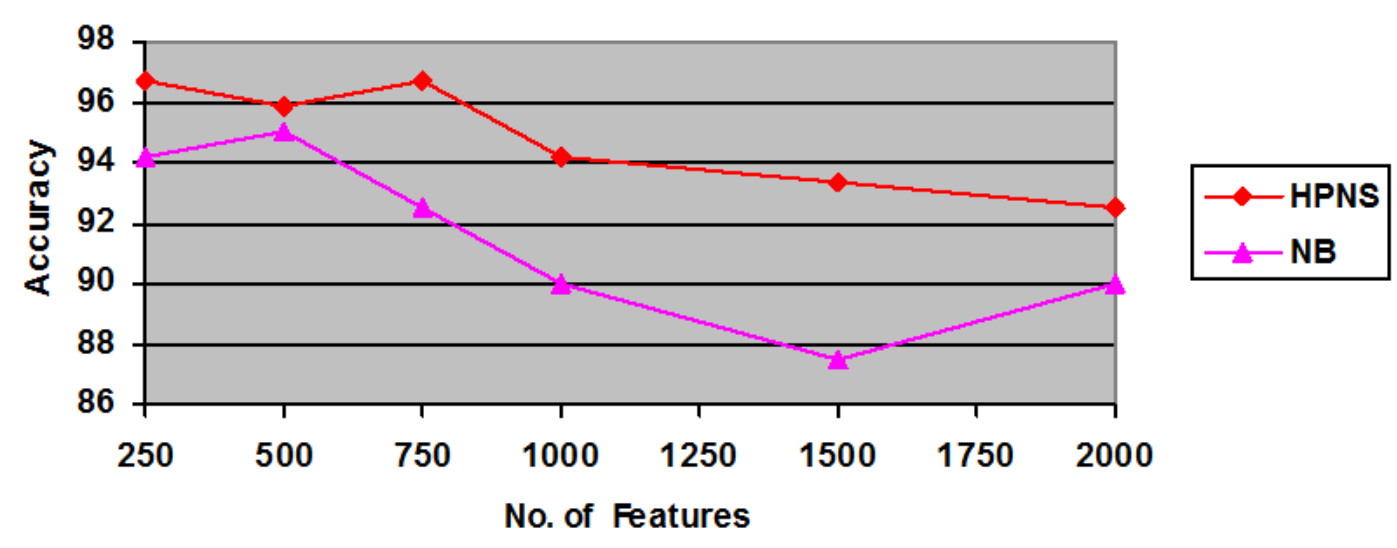

الثنكل(16) دقة المصنفات لمجموعة الموثائق الكلية

يتبين من قيم دقة المصنفات الموضحة في الجداول (2),(3),(4) أنه كلما زاد عدد الخواص كلما قلت

دقة المصنف لوثائق هندسة البرمجيات، ويقدم عدد الخواص 250 و 500 أفضل دقة تتبؤ بالنسبة للمصنفات. يعد المصنف HPNS المعتمد على تقانات المناعة التكيفية أفضل من مصنف NB؛ إذ يمتلك دقة تتبؤ عالية تصل إلى 100\% إذا تدرب على الوثائق مسبقاً و95\% لوثائق الاختبار وتكون دقته 96.67\% لمجموعة الوثائق الكلية من تدريب واختبار.

يتضمن الجدول (6) مقاييس (TP , FP , TN , FN) لوثائق الاختبار (40) وثيقة لـ (250) خاصية.

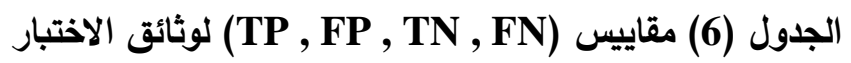

\begin{tabular}{|c|c|c|c|c|}
\hline Model & TP\% & FP\% & TN\% & FN\% \\
\hline HPNS & 95 & 5 & 95 & 5 \\
\hline NB & 90 & 10 & 90 & 10 \\
\hline
\end{tabular}

يتضمن الجدول (7) عدة مقاييس لتصنيف وثائق الاختبار بالاعتماد على قيم جدول (4). الجدول (7) عدة مقاييس لتصنيف وثائق الاختبار

\begin{tabular}{|c|c|c|c|c|c|c|}
\hline Measure & Precision & Recall & Specificity & F $_{1}$-macro & \multicolumn{2}{|c|}{ F $_{1}$-micro } \\
Model & & & & & & \\
\hline HPNS & 95 & 95 & 95 & 95 & 95 & 95 \\
\hline NB & 90 & 90 & 90 & 90 & 90 & 90 \\
\hline
\end{tabular}




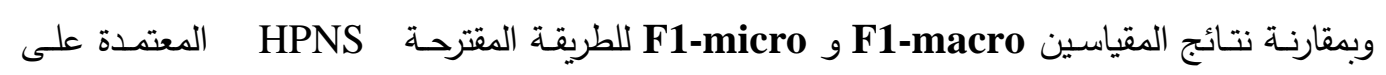

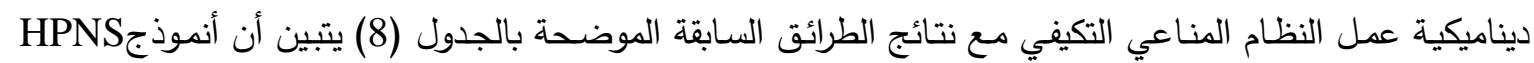
أفضل من الطرائق السابقة.

الجدول(8) مقارنة نتائج دقة مصنفات مختلفة لمقياسي F1-macroو F1-micro

\begin{tabular}{|c|c|c|c|c|}
\hline $\begin{array}{c}\text { Results reported } \\
\text { by }\end{array}$ & Dataset & $\begin{array}{c}\text { Classifier } \\
\text { Used }\end{array}$ & F1-Micro & F1-Macro \\
\hline $\begin{array}{c}\text { [Tan et al., } \\
2005] \\
{[36]}\end{array}$ & 20 Newsgroup & Centroid & 0.8420 & 0.838 \\
\hline \multirow{2}{*}{$\begin{array}{l}\text { [Qian et al., } \\
\text { 2007] } \\
{[32]}\end{array}$} & \multirow[b]{2}{*}{ Reuters 21578} & Decision Tree & 0.884 & 0.822 \\
\hline & & Linear SVM & 0.920 & 0.871 \\
\hline \multirow{4}{*}{$\begin{array}{c}\text { [Lan et al., } \\
2009] \\
{[21]}\end{array}$} & \multirow[b]{2}{*}{ Reuters 21578} & SVM & 0.921 & 0.900 \\
\hline & & K-NN & 0.840 & 0.825 \\
\hline & \multirow{2}{*}{20 Newsgroup } & SVM & 0.808 & 0.808 \\
\hline & & K-NN & 0.691 & 0.691 \\
\hline \multirow{2}{*}{$\begin{array}{c}\text { Nada and } \\
\text { [Rasha] } \\
2012 \\
\end{array}$} & \multirow{2}{*}{$\begin{array}{c}\text { Software } \\
\text { Engineering } \\
\text { Documents }\end{array}$} & HPNS & 0.95 & 0.95 \\
\hline & & NB & 0.90 & 0.90 \\
\hline
\end{tabular}

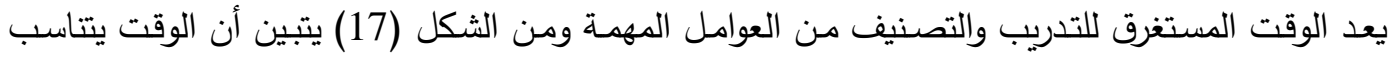

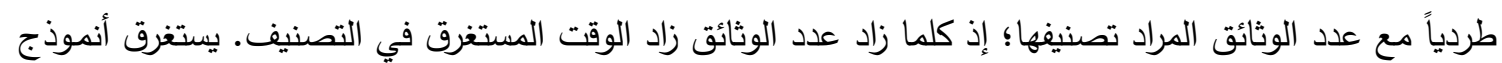

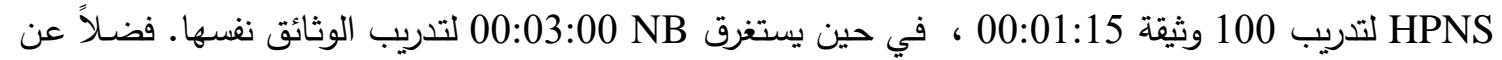

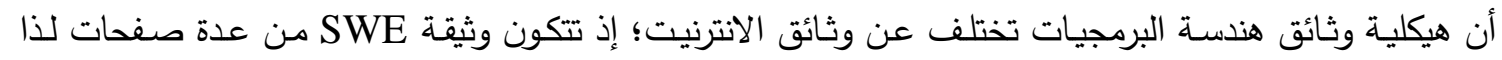

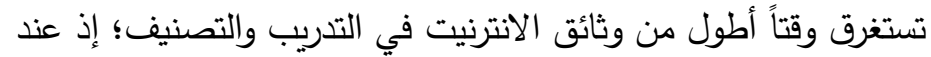

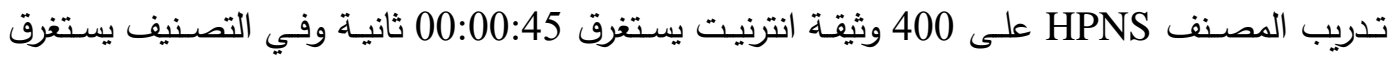

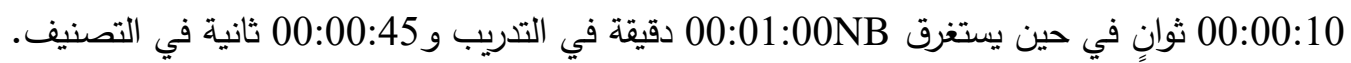

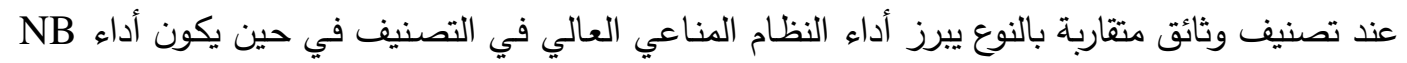

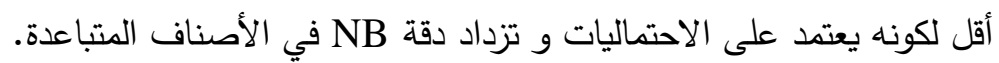




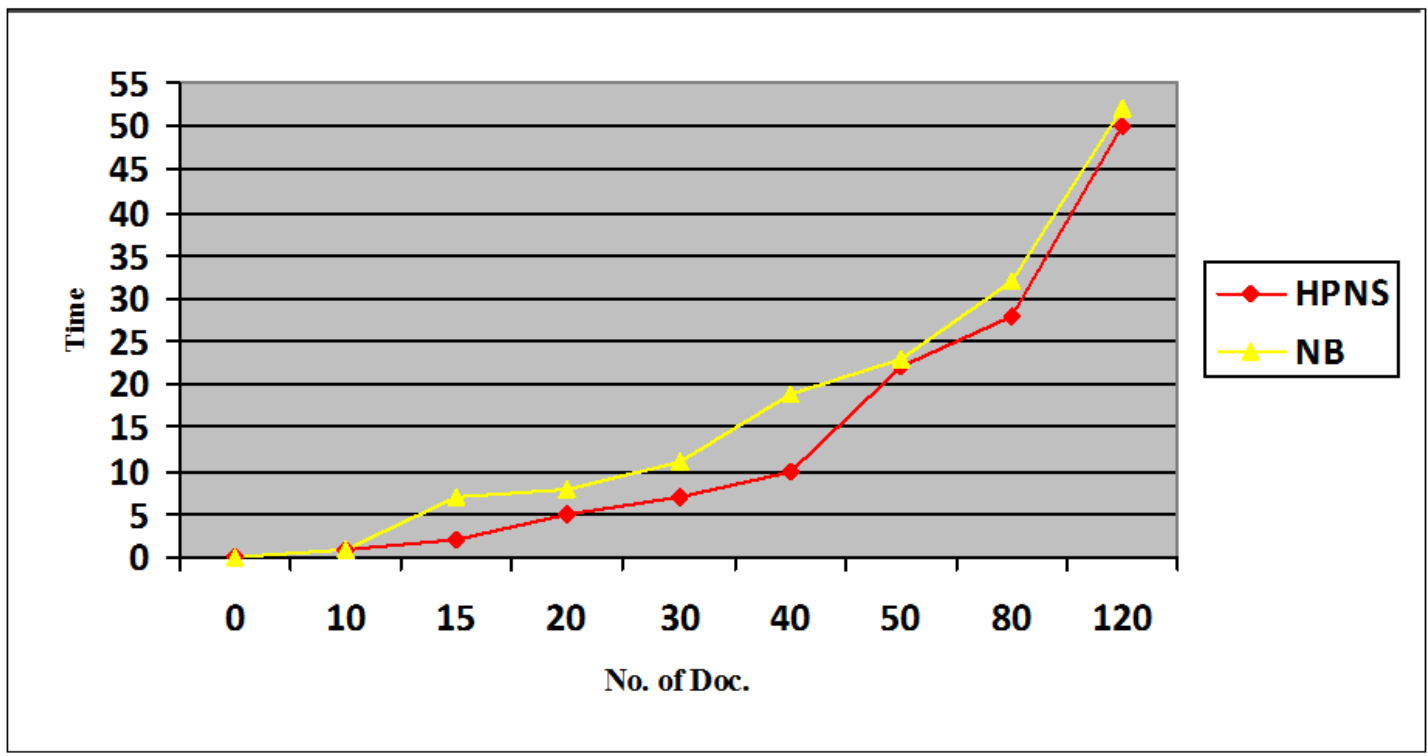

الثكل (17) علاقة الوثائق مع الوقت المستغرق لتصنيفها

من خلال تطبيق أنموذج HPNS في البحث لغرض تصنيف وثائق هندسة البرمجيات وعلى وفق النتائج

التي تم الحصول عليها، تم التوصل إلى الاستتتاجات الآتية:بعد دراسة بعض تقانات النظام المناعي AIS اخيرت

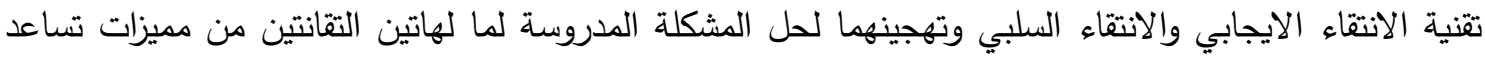
في عملية التصنيف. بعد ملاحظة نتائج البحث التي تم الحصول عليها تم التوصل إلى جدارة التطرق إلى الى التى الموضوع الذكائي الجديد وهو الأنظمة المناعية الاصطناعية. دقة نظام HPNS في تصنيف الوثائق عالية، حتى دئ

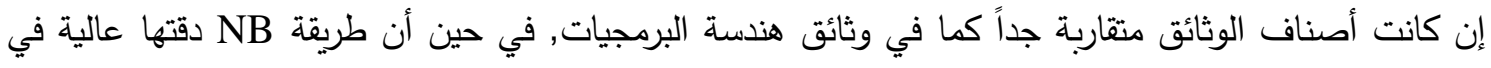

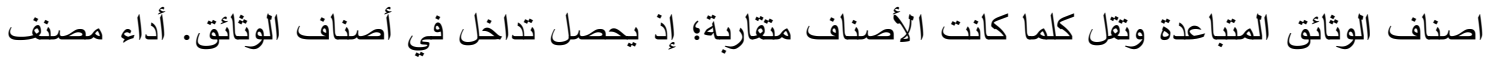

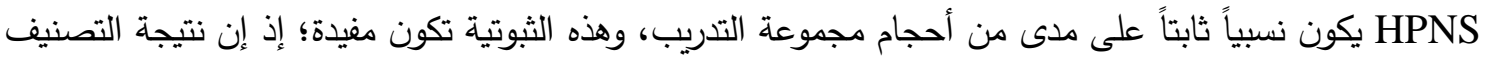

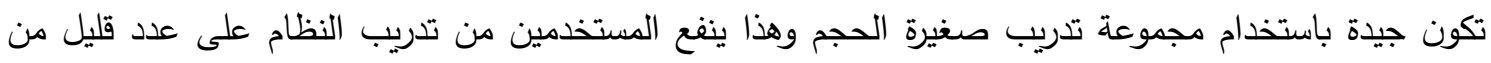
الوثائق وفي الوقت نفسه يمتلك تنبؤات دقيقة. يمتلك النظام المناعي بصورة عامة القابلية على تلى تكوين مصنفات ذات دقة تتبؤ أفضل باستخدام متجهات خواص ذات حجم أقل من متجهات خواص NB. NB. إن اختيار المصنف لا يعتمد

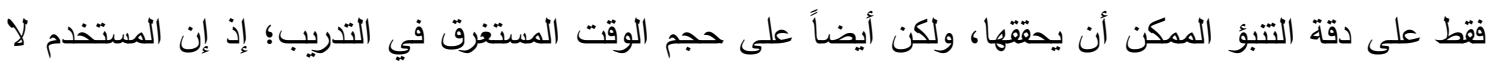

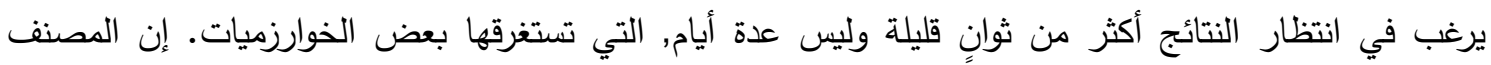
المبني على تقانة عمل النظام المناعي التكيفي يحقق دقة تتبؤ جيدة وفي وقت قليل جداً يسمح باستخدامه في مشاكل العالم الحقيقي. 


\section{المصادر}

[1] Aickelin, U., (2004), "Artificial Immune Systems (AIS) - A New Paradigm for Heuristic Decision Making", Inciter Keynote talk, Annal Operational Research conference 46, York, UK.

[2] Brownlee, J., (2008), "Clonal Selection as an Inspiration for Adaptive and Distributed Information Processing", $\mathrm{PhD}$ thesis, Swinburne University, Melbourne, Australia.

[3] Burnet, F., M., (1959), "The Clonal Selection Theory of Acquired Immunity ". Vanderbilt, University, Press, Nashville, T.N. Canada, Journal of Information Technology Education Volume 6.

[4] Cohen, W., Singer, Y., (1999), "Context sensitive learning methods for text categorization", ACM Transactions on Information Systems, vol. 17, no. 2, pp. 141- 73.

[5] De Castro L.N., Von Zuben, F.J., (2002), "Learning and optimization using the clonal selection principle", IEEE Trans. Evol. Comput., vol. 6, no. 3, pp 239251.

[6] De Castro, L. N., Timmis, J., (2002). "Artificial Immune Systems: A New Computational Intelligence Approach", ISBN 1-85233-594-7, Springer, England.

[7] Engelbrecht, Andries, P., (2007), "Computational Intelligence: An Introduction", 2nd edition, University of Pretoria, South Africa, John Wiley \& Sons Ltd.

[8] Forman, George, Eshgi, Kave, Chiocchetti, (2005), "Finding Similar Files in Large Document Repositories", Proc. $11^{\text {th }}$ ACM International Conf. on Knowledge Discovery and Data Mining (KDD'05), 21-25, Chicago, Illinois, USA

[9] Forrest, S., Perelson, A., Allen, L., Cherukuri, R., (1994), "Self-Nonself Discrimination in a Computer", Proc. of the IEEE Symposium.

[10] Fouad, Walid, A., Badr, Amr, A., Abdel- Rahman, Ebrahim, F., (2007), "A compartive Study of web document classification approaches", Proc. $37^{\text {th }}$ International Conf. on computers and industrial Engineering, pp. 197-206, Alexandria, Egypt.

[11] Goldsby, Richard, A., Kindt, Thomas, J., Osborne, Barbara, A, Kuby, J., (2003), " Immunology", $5^{\text {th }}$ Edition, W. H. Freeman and Company.

[12] Greensmith, J., Aickelin, U., (2009), "Artificial Dendritic Cells: Multi-faceted Perspectives", in Human-centric information processing through granular modeling, studies in computational intelligence (182). Springer, Berlin, pp 373395, ISBN 9783540929154.

[13] Greensmith, J.,( 2003), "New Frontiers For An Artificial Immune System", MSC Thesis, University of Leeds, Hewlett Packard Labs Technical Report Number HPL.

[14] Hotho, A., Nurnberger, A., PaaB, G., (2005), " A Brief survey of Text Mining", Journal for Computational Linguistics and Language Technology, Vol 20, pp. 1962.

[15] Ikonomakis, M., Kotsiantis, S. , Tampakas, V., (2005), "Text Classification Using Machine Learning Techniques", WSEAS Transactions on Computers, Issue 8, Volume 4, pp.966-974.

[16] Iqbal, A., (2006), "Danger Theory Metaphor In Artificial Immune System For System Call Data", PhD theises, Universiti Teknologi Malaysia.

[17] Ismail, Nabil, A., Abdul-Kader, H., Al-Sheshtawi, Khaled, A.,(2010), "Artificial Immune Clonal Selection Classification Algorithms for Classifying Malware and 
Benign Processes Using API Call Sequences", IJCSNS International Journal of Computer Science and Network Security, VOL.10 No.4, pp.31-39.

[18] Joachims, T., (1998), " Text categorization with support vector machines: Learning with many relevant features". Proc. Of European Conf. on Machine Learning (ECML), Vol 1398, pp. 137-142.

[19] Kamthan,P., (2007), "On the Prospects and Concerns of Integrating Open Source Software Environment in Software Engineering Education", Concordia University, Montreal, Quebec,

[20] Khelil, H., Benyettou, A.,(2006), Artificial Immune Systems For Illnesses D Diagnostic, Ubiquitous Computing and Communication Journal, Algeria.

[21] Kuby, J., (1997), "Immunology", 3rd Ed., W. H. Freeman and Co.

[22] Lan, M., Tan, C. L., Su. J., and Lu, Y.2009. Supervised and Traditional Term Weighting Methods for Automatic Text Categorization. IEEE Transactions on Pattern Analysis and Machine Intelligence, Volume: 31 (4), pp. 721 - 735.

[23] Matzinger, P., (1994), "Tolerance, danger and the extended family", Annual Reviews in Immunology, 12:991-1045.

[24] Meyer, B.J., Meij, H.S., Grey, S.V., Meyer, A.C., (1996), "Fisiologie van die mens -Biochemiese", fisiese en fisiologiese begrippe. Kagiso Tersier - Cape Town, $1^{\text {st }}$ Edition.

[25] Middlemiss, M., (2006), "Positive and negative selection in a Multilayer Artificial Immune System", information science paper No. 2006/03 Dunedin University of Otago, NEW ZEALANDPP. 17.

[26] Mitchell, T., (1997), "Machine Learning". McGraw Hill, New York.

[27] Mohammad, A., (2008), "Support Vector Machine Text Classification for Arabic Articles: Ant Colony Optimization Based Feature Subset Selection", PhD thesis, Arabic Academy for Banking and Financial Sciences, Amman, Jordan.

[28] Nanda, Satyasai, J., (2009), "Artificial Immune Systems: Principle, Algorithms And Applications", MSC thesis, Thapar University, India.

[29] Negi, P., (2006), "Artificial Immune System Based Urban Traffic Control", MSC thesis, A\&M University, Texas.

[30] Ojasvini, Nitesh, Piyush, Thakur, N., Rehalia, A., (2018), "Intrusion Detection System Using Artificial Immune System: A case study ", International Journals Advanced Research in computer Science and Software Engineering, ISSN: 2277$128 \mathrm{x}$, vol. 8, Isuue -2 .

[31] Onan, A., (2015), " Artificial Immune System Based Web Page Classification", In: Silhavy R., Senkerik R., Oplatkova Z., Prokopova Z., Silhavy P. (eds) $\underline{\text { Software Engineering in Intelligent Systems. Advances in Intelligent Systems and }}$ Computing, vol 349. Springer, Cham".

[32] Özgür, A., (2004), "Supervised and Unsupervised Machine Learning Techniques For Text Document Categorization", MSC thesis, , Bo gazici University.

[33] Pawar, P., Gawande, S., (2012), " A Comparative Study on Different Types of Approaches to Text Categorization", International Journal of Machine Learning and Computing, Vol. 2, No. 4.

[34] Pazzani, M., Billsus, D., (1997), "Learning and revising user profiles: the identification of interesting web site ", machine learning, pp. 313-331.

[35] Qian, T., Xiong, H., Wang, Y., and Chen, E. 2007. On the strength of hyperclique patterns for text categorization. An International Journal Information Sciences, Vol. 177, pp. 4040-4058. 
[36] Ramdane, ch., Chikhi, S., (2017), "Negative Selection Algorithm: Recent Improvements and Its Application in Intrusion Detection System", International Journal of Computing Academic Research (IJCAR), ISSN 2305-9184, vol.6, no. 2,pp. 20-30

[37] Romero, A., Niño, F., (2007), "An Artificial Immune System Based on Information Theory for Keyword Extraction from Text Documents", Revista Avances en Sistemas e Informática, Vol.4 No. 2, pp. 25-32, Edición Especial: II Congreso Colombiano de Computación - CCC.

[38] Saranya, C., Thenmozhi, D., (2015), " Machine Learning Approach to Document Classification using Concept based Features ", International Journal of Computer Applications (0975 - 8887) Volume 118 - No.20.Sciences, Amman, Jordan.

[39] Sebastiani, F., (2002), "Machine Learning in Automated Text Categorization", ACM Computing Surveys, vol. 34, no. 1, pp. 1-47.

[40] Semberecki, P., Maciejewski, H., (2017), " Deep Learning methods for Subject Text Classification of Articles", Proceedings of the Federated Conference on Computer Science and Information Systems pp. 357-360 ISSN 2300-5963 ACSIS, Vol. 11.

[41] Songbo, T., Cheng, X., Ghanem, M. M., Wnag, B., and Xu, H. 2005. A novel refinement approach for text categorization. In the Proceedings of Fourteenth ACM International Conference on Information and Knowledge Management, pp $469-476$.

[42] Tan, S., (2008), "An improved centroid classifier for text categorization". Journal of Expert System with Applications, Vol 35, pp 279 - 285.

[43] Twycross, Jamie, P., (2007)," Integrated Innate And Adaptive Artificial Immune System Applied To Process Anomaly Detection", PhD theises, University of Nottingham, UK.

[44] Wei , T., (2010), "Homology Modeling of Toll-Like Receptor Ligand-Binding Domains: A Leucine-Rich Repeat Assembly Approach",PhD theises, LudwigMaximilians-Universität München.

[45] Williams, C., Harry, R., and McLeod, J. (2007), "Mechanisms of apoptosis induced DC suppression", Journal of Immunology, 120S158. 